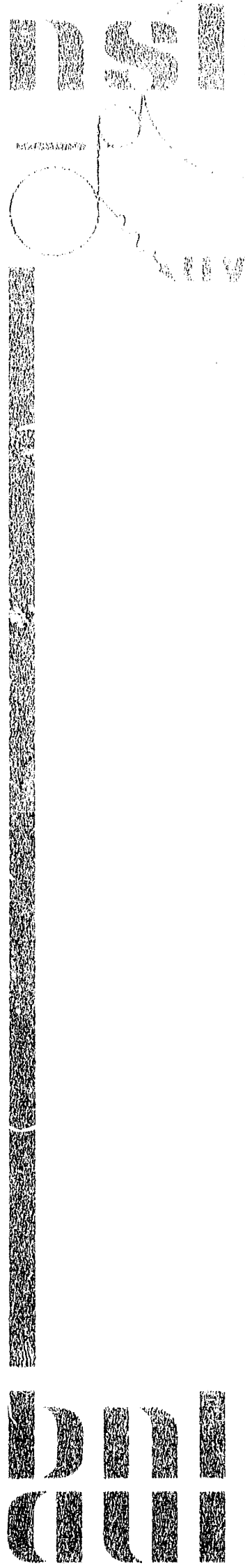

L.N. Blumberg

March 1992

\title{
NATIONAL SYNCHROTRON LIGHT SOURCE
}

BROOKHAVEN NATIONAL LABORATORY ASSOCIATED UNIVERSITIES, INC.

Under Contract NO. DE-ACO2-76CH00016 with the

UNITED STATES DEPARTMENT OF ENERGY 
as an secount of work spomsored by an ngency of the United Neither the United states (bovernment nor any ayency thereof.

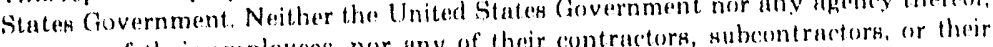

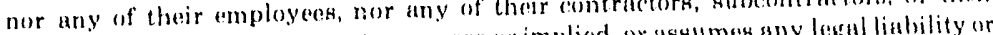

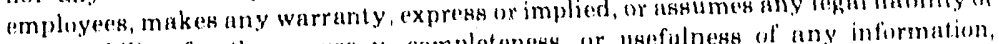
responsibility for the accuracy, completeness, or usefulnese of any informationt apparatus, product, or process diselosed, or represents that its use would mot in fing

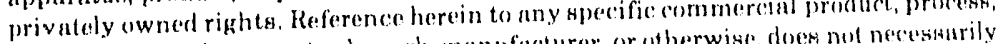
or service by trade name, trademark, manufacturer, or otherwise, doem not necesalarily or service by trade name, trademark, manufacture or or favoring hy the linitedstates constitute or imply its condorsement, recommendation, or favering hy the ling views and Government or any agency, contractor or subcontractor thereof. The vinw whe opinions of authors expressed herein do not necessarily atate of reflect hoses United stutes Government or any apency, contractor or subcontractor thereof 
BNL- -47356

DE92 013011

CONCEPTUAL DESIGN STUDY OF AN INTENSE X-RAY SOURCE FOR CORONARY ANGIOGRAPHY

\author{
L.N. Blumberg
}

March 1992

NATIONAL SYNCHROTRON LIGHT SOURCE

BROOKHAVEN NATIONL LABORATORY, ASSOCIATED UNIVERSITIES, INC. UPTON, LONG ISLAND, NEW YORK 11973

$$
\text { aldata }
$$


This work was originally completed in the spring of 1983 at the suggestion of Drs. E. Rubinstein and R. Hofstadter while the author was on leave of absence at Stanford University and SSRL. The initial studies of Digital subtraction Angiography at SSRI on phantom and animal hearts were in progress and showed encouraging results in imaging occlusions of vessels comparable in diameter to human coronary arteries. It therefore seemed an appropriate time to study the prospect of designing a dedicated compact $x-r a y$ source which could yield adequate intensity of iodine $\mathrm{K}$-edge $33.16 \mathrm{KeV} \mathrm{X}-$ Rays and be affordable for a major medical center and of sufficient small size to be located within or adjacent to such an urban setting. Subsequently, studies on human subjects were imitated at SSRL and have continued at BNL and the Deusches ElektrmenSynchrotron (DESY/ HASYLAB) NIKOS project, Hamburg, Germany. Initial results from these studies are encouraging - the image quality is now adequate to resolve major arterial blockages although not yet of comparable quality to replace the existing invasive catheterization method for clinical applications. Concurrently, additional studies of suitable x-ray sources have been pursued in the U.S., Germany and Japan, and renewed interest has recently been evident at the Natural Institute of Health (NHLBI) to develop such sources. It therefore seems appropriate at this time to reissue this stanford (HEPL) report as a BNL report to provide wider distribution of the (unpublished) study.

L. Blumberg, March 1992 


\begin{abstract}
. . . . . . . . . . . . . . . . . 1
I. INTRODUCTION AND ANALYSIS . . . . . . . . . . . 2

II. RESULTS . . . . . . . . . . . . . . . . . 9

III. DISCUSSION . . . . . . . . . . . . . 16

IV. PRELIMINARY COST ESTTMATE . . . . . . . . . . . 20

V. ACKNOWLEDGEMENTS . . . . . . . . . . . . . 22

REFERENCES . . . . . . . . . . . . . . . . 23

TABLES AND FIGURES . . . . . . . . . . . . 25
\end{abstract}


Calculations are presented for several $1.4 \mathrm{GeV}$ electron storage ring designs which, with an ultra-h1gh fleld ( $80 \mathrm{kG}$ ) superconducting wiggler magnet and beam current $I=400 \mathrm{~mA}$, w111 generate $33.16 \mathrm{keV} x-\mathrm{ray}$ beam at 20 m from the wiggler of adequate intensity $\left(610^{9} / \mathrm{mm}^{-2} \mathrm{sec}^{-1}\right)$ and areal size for lodine K-edge coronary dichromography in humans. The preferred design is a 54 m clrcumference, 4 superperlod ring with 3.6 m long atralght sections for wigglers, 0.7 m stralght sections for injection klckers, RF cavities, harmonic cavities and Eeedback devices, and $0.46 \mathrm{motratght}$ sections for chromat1c1ty-correcting sextupoles $S_{d}$ and $S_{F}$. Bending magnets are short $(0.6 \mathrm{~m}) 15^{\circ}$ bend "window frame" conventiona! dipoles operated at high (20 kG) fleld. A $500 \mathrm{MHz}$ RF syotem 18 proposed to obtain a high harmonic number, $h$ 90 , and large peak RF voltage $V_{m}-2.4 \mathrm{MV}$ to opt1mize the Touschek 11fetime at infection energy. Infection with a 100 MeV Mlcrotron 18 proposed without use of an Interpediate Booster synchrotron. The gas scattering lifetime at $100 \mathrm{MeV}$ and $10^{-10}$ Torr, $\tau_{c}=46 \mathrm{sec}$, 18 sdequate and the transverse damping tlue $T_{x}=0.63$ sec with the additional damping provided by the wiggler is sufficlently small. The bremstrahlung lifetime $T_{B} 1 s$ more than adequate; however, the Touschek lifetime without $a$ wiggler 18 only it $=0.37$ sec at $100 \mathrm{MeV}$ but can be inceased to - $15 \mathrm{sec}$ if the wiggler $1 \mathrm{~s}$ used to increase damped emittance and thus beam size. The Touschek ilfetime for the assumed $400 \mathrm{~mA}$ beam appears to be the 11witing factor for low energy injection. In the present study we have nut addressed the photon beam line design problema posed by the $50.8 \mathrm{kw}$ power output of the wiggler, nor have tracking calculations been performed to determine the decrease in dynamic aperture when the sextupoles are energized. A preliminary cost estlmate 1 s presented based on an extrapolation from existing or contemplated machines in the same energy range. Without a Booster ring, wiggler and beam 11 ne, and bullding for support staff (but including shlelding) the overall faclitey cost 1 s $\$ 7.35 \mathrm{M}$. 


\section{Introduction and Analysis}

We anticipate that a clinical demonstiation of non-invasive digital subtraction coronary angiography (DSA) with tunable $x$-rays (dichromography) will create a need for many tunable $x$-ray sources that are intense, affordable and compact. These sources will be required near to urban medical centers throughout the world and therefore readily accessible to the patient population and to the medical community, specifically, the dichromographic method under development at stanford, involving the subtraction of digital $x$ ray images obtained at energies immediately above and below the iodine Kabsorption edge ( $33.16 \mathrm{keV}$ ) uses radiation from a wiggler beam at the electron storage ring SPEAR at the stanford Synchrotron Radiation Laboratory. The $33.16 \mathrm{keV}$ photon intensity from the 8 -pole, $18 \mathrm{~kg}$ peak field wiggler magnet in beam line IV, with SPEAR operating at $3 \mathrm{GeV}$ electron energy and $100 \mathrm{~mA}$ of circulating electron beam, is about $6 \times 10^{9}$ photons $\mathrm{mm}^{-3} \mathrm{sec}^{-1}$ in the forward direction at $20 \mathrm{~m}$ in a photon energy interval of $17 \mathrm{eV}$ cobtained by Bragg scattering on a si(220) crystal'). After passage through $=20 \mathrm{~cm}$ of tissue in a 5 msec exposure a flux of about $3.5 \times 10^{4}$ photons $\mathrm{inm}^{-2}$ is incident on si(Li) detector and provides a signal-to-noise ratio of about 7.5 in a $0.5 \times 0.5 \mathrm{~mm}^{2}$ pixel for an iodine arterial thickness of $2.5 \mathrm{mg} \mathrm{cm} \mathrm{cm}^{-2}$.

In the present study we have adopted the above intensity as a design criterion, although we note that the intensity desired by the Hamburg groupj, $4.0 \times 10^{10} \mathrm{~mm}^{-2} \mathrm{sec}^{-1}$, is somewhat higher. Also, in the present context, we do not regard the size of the SPEAR machine (circumference $C=234 \mathrm{~m}$ ) or other recentiy completed high energy synchrotron radiation sources, as small. These include the Brookhaven (NSLS) $2.5 \mathrm{GeV} X-\mathrm{ray}$ ring" ( $C=170 \mathrm{~m}$ ), the Daresbury $(U . K) .2.0 \mathrm{GeV} S R S^{5}(C=96 \mathrm{~m})$, ard the KEK (Japan) $2.5 \mathrm{GeV} P F^{6}(C=178 \mathrm{~m})$.

The following considerations have guided the present study.

As emall a ring as possible with conventional magnets is required. This implies that we choose a bending magnet with the highest field practicable. We therefore choose a window frame design. Such magnets have operated in high energy particle spectrometers at $20 \mathrm{kG}$. With field tolerance comparable to the uniformity required in storage rings; about 1 part in 10". We are particularly encouraged in this choice by the recent successful operation of the SLAC Damping Ring' using magnets of similar design and strength. A consequence of small machine size is that we choose the lowest electron energy consistent with the required photon intensity. Since we plan to use a wiggler source, the photon intensity is also a function of the peak wiggler field. The photon spectrum from a wiggler is completely characterized by the critical energy

$$
\varepsilon_{c}=0.665 B_{0}(T) E_{G}^{2}\left(G e V^{2}\right) \mathrm{ke}:
$$

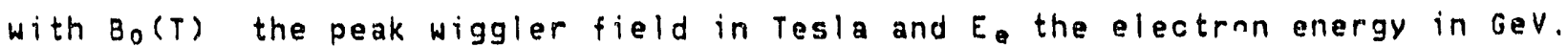
We desire the strongest wiggler field possible (to minimize the beam energy) that will yield a critical energy near the value $\varepsilon_{c}=10.77 \mathrm{keV}$ of the SSRL wiggler. In this regard we make an extrapolation and choose a wiggler with superconducting coils and a peak field of $B_{0}=80 \mathrm{kG}$. The first superconducting wiggler fabricated at $B N L$ yielded $B_{0}=60 \mathrm{kG}$, and improvements in its design to better constrain the movement of the superconducting braid are readily envisioned. B Thus, 80 kG seems a possible goal in this technology. In Fig. (1) we show the $33.16 \mathrm{keV}$ photon intensity from such a device at 
various electron energies compared to the $60 \mathrm{kO}$ NSLS wiggler and the $18 \mathrm{kG}$ SSRL. wiggler. The curves were calculated using the expression given by oreen'

$$
\frac{d N}{d t}=\frac{3.69465 \times 10^{11} \Delta \varepsilon(e V) \Delta \theta(m r) I(m A) N_{p}}{B(k G) E_{e}(\mathrm{GeV})} \int_{\varepsilon / \varepsilon_{c}}^{\infty} K_{5 / 3}(y) d y
$$

where $\Delta \theta(m r)$ is the angle increment in the plane of the bend in milliradians, $\Delta \varepsilon(e V)$ is the photon energy increment in electron volts at energy $\varepsilon$, I (mA) is the circulating electron beam current in the ring in milliamps. $N_{p}$ is the number of poles of the wiggler, $K_{5} / 3$ is the modified Bessel function of the secrsnd $k$ ind, and the field is obtained from the assumption that the electron trajectory and the magnetic field in the wiggler are sinusoidal

$$
x=x_{0} \sin \left(2 \pi z / \lambda_{0}\right) \quad B=B_{0} \sin \left(2 \pi z / \lambda_{0}\right)
$$

$\lambda_{0}$ is the length of the wiggler period, and the amplitude of the oscillation $x_{0}$ is given in terms of the wiggler strength parameter $K a s^{10}$

$$
x_{0}=(k / y)^{2} p_{0}
$$

with $\gamma$ the electron energy in units of $m_{0} c^{2}, i, a, \quad \gamma=E_{e} / m_{0} c^{2}$, $\rho_{0}$ the bending radius of the electron at the point where the wiggler field is $B_{0}$, and Bopo $=33.35 \mathrm{E} .(\mathrm{GeV})$. The wiggler strength parameter (dimensionless) is $K \simeq 0.934 \lambda_{0}(\mathrm{~cm}) B(T)$. The wiggler wavelength chosen for Fig. (1) is $\lambda_{0}=17.4 \mathrm{~cm}$ and the angle is given by:

$$
\tan \theta=\frac{d x}{d z}=\left(\frac{2 \pi x_{0}}{\lambda_{0}}\right) \cos \left(\frac{2 \pi z}{\lambda_{0}}\right)
$$

For our choice of field and $\lambda_{0}, K=130$. We choose an energy for the medical $x$-ray source of $E_{0}=1.4$ GeV on the basis of these results. The angular distributions for the lower energy rings in fig. (1) are broader than for the NSLS or SSRL sources since the maximum angle from $E q$. (5) is $(K / y)$. For our choice of energy the full width of the radiation cone at half intensity is $\Delta \theta=60 \mathrm{mrad}$. The peak intensity is seen to be down by a factor of 2 from the SSRL case, but it is clear fror, the performance of present day storage rings with low longitudinal impedance (smooth vacuum chamber) that the ring current will exceed the SPEAR value of $100 \mathrm{~mA}$ by at least a factor of 2. The Daresbury SRS has attained'l $I_{\max }=600 \mathrm{~mA}$, the $K E K(J a p a n)$ PF group reports'2 $I_{\max }=200 \mathrm{~mA}$ (after vacuum system bakeout and argon discharge cleaning), and the NSLS VUV ring's has reached 1 max $=400 \mathrm{~mA}$ without the use of any of several means of damping instabilities or of eliminating the sources which excite the instabilities. In Fig. (2) we show the wiggler photon spectrum for the $9.4 \mathrm{GeV}$ machine from Eq. (2) compared to the NSLS and SSzL sources. This shows that for the SSRL wiggler and the high field wiggler of the present 
design, the $33.16 \mathrm{keV} x$-rays are well above the critioal energy $\varepsilon_{G}$ and are in the portion of the spectrum where the intensity is decreasing exponentialiy

$$
\left(\frac{d^{2} I}{d(u d \Omega 2}\right)_{\theta=0^{\circ}} \approx \frac{3 e^{2}}{2 \pi c} \gamma^{2}\left(\begin{array}{l}
\omega \\
i i{ }_{c}
\end{array}\right) e^{\left.-2(u) /()_{c}\right)}
$$

Eq. (6) gives the energy per turn radiated by an electron in a frequency interval du and into solid angle $d \Omega$. If we divide by the energy per photon tis and note that $e^{2} /$ tic $=a \equiv 1 / 137$ and multiply by the number of electrons per second I(amps)/e(coulombs) and the fraction $(\Delta \theta) / 2 \pi$ of electrons in an incrament $\Delta \theta$, then Eq. (6) is a good approximation to our Eq. (2).

The ring should contain more than one wiggler straight section. It seems unreasonable to limit the machine capability to one beam line (although a 60 mrad beam could be split into several, perhaps five, beams for simultaneous patient use). The incremental cost of adding straisht sections is small in comparison to the total machine cost, since the same number of magnets and power supplies would be required in the storage ring and the same investment in pre-injector accelerator(s). conventional facilities, real estate and foundations are involved. We choose a 4-superperiod machine with four long straight sections; three for wigglers and one for injection magnets.

The length of the long straight sections should be adequate to accommodate bump magnets at either end of the wiggler to provide a local orbit deformation in the event that the method of sweeping the electron beam vertically in the wiggler's to move the horizontal fan of synchrotron radiation across the area of interest should prove feasible. As presently envisioned, the bumps would consist of four dipoles, two at either end of the wiggler and powered by a common pulsed capacitor discharge power supply with full sine wave excitation. Approximately one meter would be required at either end of the wiggler to provide the $=5 \mathrm{mrad}$ beam deflection. Since the present 3-periad, $\lambda_{0}=17.4 \mathrm{~cm}$ BNL superconducting wiggler 16 requires $1.4 \mathrm{~m}$ of straight section length, we estimate that the 5-period, $\lambda_{0}=17.4 \mathrm{~cm}$ wiggler contemplated here will at least use $1.8 \mathrm{~m}$. Thus, the long straight section should be $=3.8 \mathrm{~m}$.

The $x$-ray beam should be removed from the machine as soon as possible since the radiation fan of $60 \mathrm{mrad}$ divergence is already $8.4 \mathrm{~cm}$ wide at the end of the straight section. This implies that there should be no more than a quadrupole singlet at the end of the straight section, followed by a speci $c$-type bending magnet to allow the photon beam to be extracted from the ring. The quadrupole should be of the horizontally converging ( $F$ ) type since we desire a large and parallel horizontal beam (high horizontal B-function) but small vertical beam (low vertical B-function) in the straight section. The latter requirement is a consequence of our choice of a horizontally bending. small vertical gap wiggler, and the theorem't that the smallest size beam one can have in a given length $l$ occurs when $B_{0}=l / 2$ at the center of the aperture. This can be seen by noting that the $B$ function in the straight section is

$$
B(s)=B_{0}+s^{2} / B_{0}
$$


and $d B / d s=0$ at $B_{0}=s=l / 2$. Thus we desire $B_{0}(v)=0.75 \mathrm{~m}$. Another, and perhaps more compelling reason for small $\beta_{v}$ at the wiggler, is the beam lifetime from gas soattering. Electrons are removed from the beam either by coulomb soattering and subsequently striking an aperture as a result of their angle deflection, or by inelastic scattering from the gas nuclet with emission of bremsstrahlung and subsequent energy loss sufficient to remove them from the stable RF bucket. If the oross seotion for the electron removal process is o then the rate of partiole loss is

$$
d N / d t=-N o n o
$$

where $c=$ veloctty of light $=2.99791010 \mathrm{~cm} / \mathrm{sec}, \quad n=$ density of gas nuclei, and $N=$ number of electrons in the ring. Eq. (8) leads to the le lifetime of the beam of

$$
r=1 / n 00
$$

For coulomb scattering we can integrate the Rutherford differential orosssection

$$
\left(\frac{d J}{d S \Omega}\right)_{R}=\left(\frac{2 a^{2}}{2 m c^{2}}\right)^{2} \frac{1}{\sin ^{4}(\theta / 2)}
$$

from $\theta=\pi$ to some angle $\theta_{m i n}$ such that electrons scattering with angles $\theta<\theta_{\text {min }}$ remain in the machine. We obtain

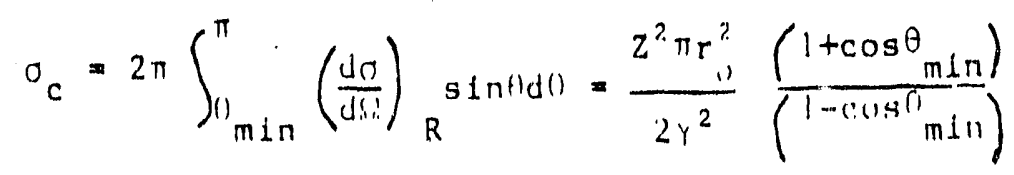

where $m=m_{0} c^{2} y$ has been used and $r_{0}=e^{2} / m_{0} c^{2}=2.81 \times 10^{-13}$ om is the classical electron radius. Using a small angle approximation for $\cos \theta_{m}$ in. we obtain $\sigma_{c}=2 z^{2} \pi r_{0}^{2} / \gamma^{2} \theta^{2} m i n$. A particle starting with an angle $\theta_{m i n}$ will undergo betatron osoillations of amplitudelo

$$
x=\theta_{m} \operatorname{in} \sqrt{\beta_{s} \beta} \sin \psi
$$

where $\psi$ is the betatron phase angle, $B_{s}$ the beta function at the scattering point and $\beta$ at the azimuth $\phi$. From $\varepsilon q$. (12) the aperture $x=a$ will occur at

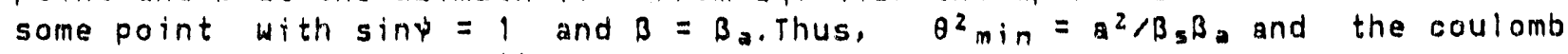
lifetime from Eq. (9) is 19

$$
\tau_{c} \simeq \frac{r^{2} a^{2}}{2 z^{2} n c \pi r_{0}^{2} \beta_{s} B}
$$


It is customary to replace the $\beta$ value at the scattering point by the average $\beta_{s}=\langle\beta\rangle$ in the ring. The point of the present discussion is that we want small $B_{\text {a }}$ at the wiggler so that the wiggler gap a can be small and the field thereby large.

The beam lifetime should be as large as possible. Another faotor affecting beam lifetime is bremsstrahlung production as noted above. The oross-section for this process is given oy Bethe and Ashk $i^{20}$

$$
d \sigma_{B}=4 z^{2} \alpha r_{\sigma}^{2} \frac{d E}{\varepsilon}\left\{\left[1+\left(\frac{E}{E_{e}}\right)^{2}-\frac{2}{3}\left(\frac{E}{E_{e}}\right)\right] \ln \left(183 / z^{1 / 3}\right)+\frac{1}{9}\left(\frac{E}{E_{e}}\right)\right\}
$$

(for complete screening) with $E=\varepsilon_{0}-\dot{\varepsilon}, \varepsilon=$ photon energy, and where $x_{0}$ is the radiation length given by

$$
x_{0}=\left[4 \pi a r_{0}^{2} z(z+\xi) \ln \left(\frac{183}{z^{1 / 3}}\right)\right]^{-1}
$$

The total cross-section for electron loss via bremsstrahlung emission can be obtained by integrating Eq. (14) over photon energy from the electron energy E. to the maximum energy size $\Delta E_{r f}$ of the RF bucket

$$
\begin{aligned}
& \sigma_{B}=\frac{1}{X_{0}^{n}} \frac{4}{3}\left\{\ln \left(\frac{E_{e}}{\Delta E_{R F}}\right)-\frac{\left(E_{e}-\Delta E_{R F}\right)}{E_{e}}+\frac{3}{8}\left(\frac{E_{e}{ }^{2}-\Delta E_{R F}^{2}}{E_{e}^{2}}\right\}\right. \\
& \cong \frac{16}{3} z^{2} a r_{0}^{2} \ln \left(\frac{183}{Z^{2} / 3}\right)\left\{\ln \left(\frac{E}{\sqrt{E_{R F}}}\right)-\frac{5}{8}\right\}
\end{aligned}
$$

In Eq. (16), $\alpha$ is the fine struoture constant and $r_{0}$ is the classical electran radius as previously defined. Also we have made the obvious approximation that E. i) $\Delta E_{r f}$. The RF buoket size is given by

$$
\Delta E_{R F}=\sqrt{\frac{2 e V_{m}^{E} e}{\pi h a_{p}}}
$$

whore $V_{m}$ is the peak RF voltage across the accelerating cavity, $h$ = harmonic number of ring = number of $R F$ wavelengths in the circumference $L$ of the synohronous particle of energy $E_{a}$, and $a_{p}$ is the momentum compaction factor of 
the ring defined as

$$
\alpha_{p} \equiv(\Delta L / L) /(\Delta p / p e)
$$

obviously we cannot evaluate $\Delta E_{r}$ and thus $\sigma_{b}$ and $\tau_{b}$ until we know the design values for the ring but, as an example, the values for SPEAR are $\alpha_{p}=0.0418$ and $h=280$ with $V_{m}=2.4 M V .21$ Thus, at $E_{0}=3 \mathrm{GeV}, \Delta \varepsilon_{r f}=19.79 \mathrm{MeV}$.

Can we eliminate the need for a Booster synchrotron? This would clearly represent a significant oost saving and make the facility more rellable. When we note the choices made by ather laboratories, we see that Daresbury and Brookhaven use Boosters with approximately $1 / 3$ the full energy of the storage ring and the KEK machine (as well as the SLAC storage rings) use full energy injection from a Linac. The Hefei (China) group plans22 $200 \mathrm{MeV}$ injection into an $800 \mathrm{MeV}$ ring, and the University of Wisconsin. SRC will injeot at $100 \mathrm{MeV}$ into the $1 \mathrm{GeV}$ ring ALLAOIN. The recent sucoess in beam storage and acceleration at Wisconsin 23 encourages us to look carefully at the possibility of a $100 \mathrm{MeV}$ microtron lijector directly into our $1.4 \mathrm{GeV}$ ring. There are three significant factors to be evaluated: gas scattering as mentioned in (4) above which has an energy dependence of $y^{2}$, the transverse and longitudinal damping times ${ }^{44}$ which vary as $1 / y^{3}$, and the Touschek lifetime 25 which incraases as $\boldsymbol{\gamma}^{5}$. Damping times will be considered when we discuss machine designs and, in particular, we will show that the presence of wigglers can have important beneficial effeots in reducing them at low energy. For the Touschek effect we follow the farmulation of Wiedemann 26 and write for the Touschek lifetime

$$
\tau_{\text {tou }} \simeq \frac{v\left(\Delta p_{m}\right)^{2}}{4 \pi r_{0}^{2} c \otimes N_{0}}
$$

where $N_{0}$ is the number of electrons per bunch, $V$ is the volume of the bunch which, for present purpuses, we take as $8 \sigma_{x} \sigma_{y} \sigma_{z}$ with $\sigma_{x}$ and $\sigma_{y}$ the standard deviation of the assumed Gaussian transverse profile and $\sigma_{z}$ the longitudinal standard deviation. $\Delta p_{m}$ is the momentum acceptance of the ring in units of moc, which if we neglect the electron rest energy and set $\beta=v / c=1$, is just $\Delta y$. the energy extent $\varepsilon_{r f}$ of the RF bucket of Eq. (17) if that quantity is expressed in units of moc ${ }^{2}$. The quantity $\mathcal{\theta}$ is a dimensionless integral given on fig. (4) and parameterized in terms of $n \equiv\left(\Delta p_{m} / \psi_{0}\right)$ and $\sigma_{p}$ the standard deviation of the electron momentum distribution in units of moc. Wiedemann shows that to good approxination $\mathcal{H}$ is a function of $n / \sigma_{p}$. However, we will want to look carefully at $\tau$ tou at low ( $100 \mathrm{MeV})$ injection energy where $\sigma_{p}$ is small. We have, therefore, calculated the integral for various $\pi$ and $\sigma_{p}$ in Fig. (4). With regard to the $\gamma^{5}$ energy dependence of $r$ tou mentioned earlier, this can be seen from Eq. (19) and the dependence $27 \sigma_{x}, \sigma_{y}$, and $\sigma_{z} \sim \gamma_{0}$ and $\Delta p_{m} \sim \gamma_{e}$ if we assume that $\eta=$ constant, i.e. that the accelerating voltage $v_{m}$ in Eq. (17) is always adjusted so that the energy acceptance is the maximum allowed by the vacuum chamber where the dispersion function $n_{x}$ of the machine is maximum. Again. we cannot evaluate $r$ tou without a specific machine design and, as with the damping time, the presence of wigglers and our ability to set a non-zero value of the dispersion function $n_{x}$ at the wiggler position, and ihus increase the beam size can have a beneficial effeot on the Touschek 
lifetime at injection energy. It should slso be pointed out that the bremsstrahiung iffetime dependence on $V_{m}$ obtained from Eqs. (16) and (17), as WEll as the Touschek lifetime dependence on bucket size $\left(\Delta p_{m}\right)^{2}$ and particles par bunch $1 / N_{0}$, both argue for high RF frequency. However, high harmonic liumber $h$ resulting from high $R F$ frequency supports the opposite conclusion. We can have both high $f_{r} f$ and low $h$ if the ring circumference is small. As a numerical example of the Touschek effect for the $700 \mathrm{MeV}$ NSLS VUV ring we have $\gamma_{0}=1369.85, \quad \eta=0.0267, \quad \sigma_{p}=4 \times 10^{-4} \gamma_{0}, \quad \sigma_{z}=3.84 \mathrm{~cm}, \quad \sigma_{x}=066 \mathrm{~cm}$, $\sigma_{y}=0.0066 \mathrm{~cm}\left(10 \%\right.$ coupling), and $N_{0}=1.1 \times 1011$ electrons/bunch. From fig. (4) $\mathcal{Q}=0.58$ and $\tau_{t o u}=9426 \mathrm{sec}$. In practice, accelerator designers do not accept the lumerical result of Eq. (19) literally since it does not contain many effects such as, increase in bunch size due to space charge effects, trapped ions in the vacuum chamber, instabilities, etc. In any case $r$ tou incrases with time as $N_{0}$ decreases. A factor of ignorance of at least two is applied.

Since we have chosen a window-frame bending magnet to obtain maximum field, it folluws that the magnet must be straight unless we select a design (solid core) which can be disassembled to install the vacuum chamber and coils. It would be undesirable to build a solid-core magnet since this would limit the rate at which the magnet could be ramped, due to Eddy currents, for the noBooster option. In the present design we will assume that the magnets have laminated cores and are straight. The penalty for this choice is that we must then allow aperture for the curvature of the beam.

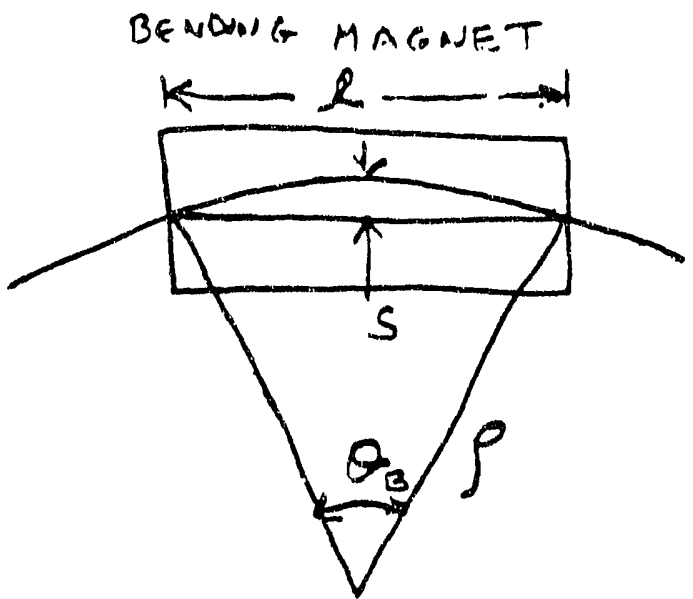


For a magnet of bending radius $\rho$ and bend angle $\theta_{B}$ the departure of the particle trajectory from a straight line, usually called the sagitta 5 (see above sketch) is

$$
S=\rho\left(1-\cos \frac{\theta_{B}}{2}\right)
$$

where can be evaluated from the magnetic rigidity $R=B \rho=33.35 E$ (GeV) kG m. For our case with $B=20 \mathrm{kG}$ and $E=1.4 \mathrm{GeV} B \rho=46.69897 \mathrm{kG} \mathrm{m}$ and $\rho=2.33495 \mathrm{~m}$. The effective length of the magnet is then $l=\rho \theta$. The sagitta for several designs we have considered is $s\left(\theta_{b}=9^{\circ}\right)=0.72 \mathrm{~cm}, \quad S\left(\theta_{b}=11.25^{\circ}\right)=1.12 \mathrm{~cm}$ and $S\left(\theta_{b}=15^{\circ}\right)=2.0 \mathrm{~cm}$. Although we do not presently know the aperture requirement of the magnet vacuum chamber, a good estimate would be the NSLS chamber of $8 \mathrm{~cm}$ width. It then seems unwise to exceed $\theta_{b}=15^{\circ}$ for the bend angle. Therefore, we have limited our lattice search to $\theta_{b} \leqslant 15^{\circ}$. Also, the special c-magnet immediately downstream of the wiggler straight will be limited to $B=15 \mathrm{kG}$ in accord with NSLS experience.28 This implies that the c-magnet will be $4 / 3$ the length of the window-frame magnet. It should also be noted that the present design is not limited to $1.4 \mathrm{GeV}$ should the wiggler fail to attain $80 \mathrm{~kg}$; we can increase the bending magnet field beyond $20 \mathrm{~kg}$ if a high quality steel such as VITRENAMEL is used. In Fig. (3) We show data of Allinger and Jackson 29 on $B$ vs H. If, for example, we consider a conductor of $2.5 \mathrm{~cm}$ width $x 5 \mathrm{~cm} \mathrm{high} \mathrm{at}$ $15 \mathrm{KA} \mathrm{cm}^{-2}$ and neglect $H$ in the core we have $H=3.75 \times 10^{6} \mathrm{Amp} / \mathrm{m}^{-1}$ $(B=47.124 \mathrm{kG})$ in the gap. From Fig. (3) this gives $H=26000$ oersteds or permeability $\mu=1.3$. Thus, the steel is not saturated even at this hish field.

To summarize the above, we have studied lattices of 4-superperiods, 4 long $(3.8 \mathrm{~m})$ straight sections at $1.4 \mathrm{GeV}$ and present some of the more favorable designs for $\theta_{b}=11.25^{\circ}$ and $15^{\circ}$ dipoles. The lattice design code COMFORTS 30 which is an improved version of the SLAC storage ring design code MAGICJ1 and the lattice design code LAMPJ2 were used. An RF frequency of 500 Mhz was assumed and peak RF voltage of $2.4 \mathrm{MV}$.

\section{Results}

A. Lattices with $15^{\circ}$ Dipoles, $L=50.96 \mathrm{~m}, h=85$

The smallest machine considered in this study is sketched below. The effective length of the wiggler is taken as $1 \mathrm{~m}$ and the quadrupoles are assumed to be $8 \mathrm{~cm}$

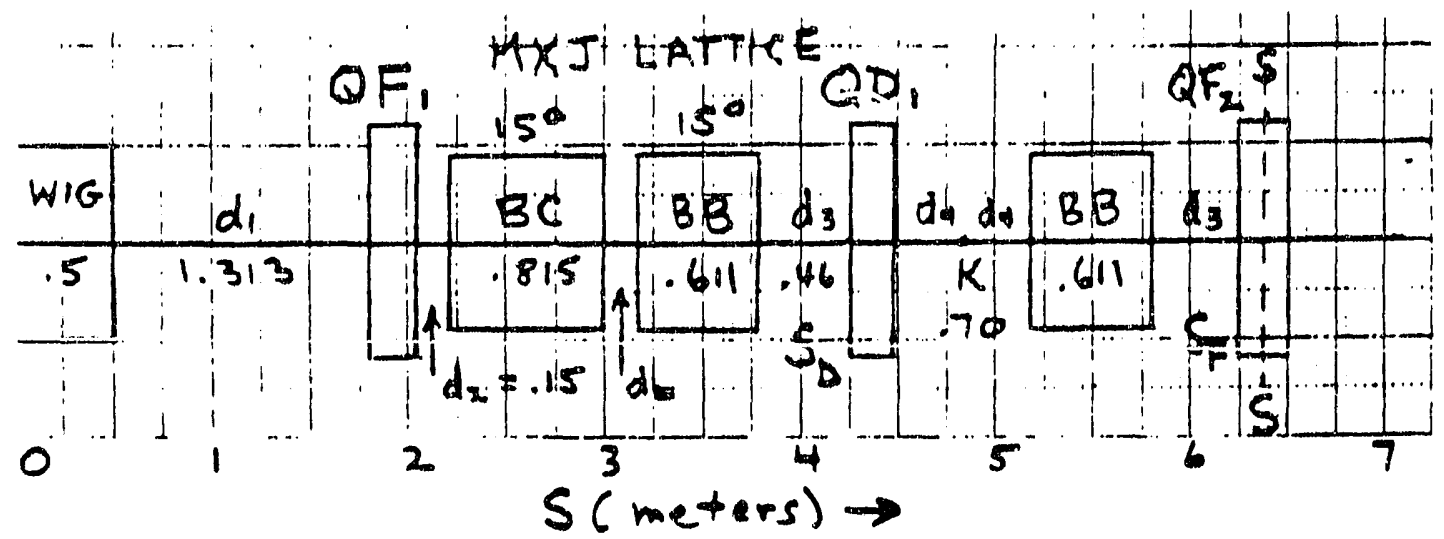


aperture and $24 \mathrm{~cm}$ effective length. The $70 \mathrm{~cm}$ straight section centered at $k$ is allowed for the injection kicker cone either side of the injection straight section). The remaining six $70 \mathrm{~cm}$ straight sectiois are sor RF cavities (2 assumed), stripline beam detector (1), harmonic RF cavity for bunch lengthening (1), longitudinal tume splitting or feedback cavity (1), and transverse feedback deflection plates (1). We assume that there will be additional space in one or more of these shoit straights inr beam current transformer. The beam position monicors are assumed to be of the $1 \mathrm{~cm}$ diameter capacitive pick-ups which have been used successfully at SLAC and aNL. and can fit under the coils of the quadrupoles. We also assume that trim windings about the quadrupole poles such as are used successfully at SPEAR will suffice for closed orbit correction. The d $=46 \mathrm{~cm}$ straights are for correction sextupoles $S_{d}$ and $S_{f}$ which are required to correct the natural chromaticity from negative values to a value +3 as required to suppress the head-tail transverse instability. $v_{x}$ and $v_{y}$ are the horizontal and vertical "tune" of the machine definedlo as $v=(2 \pi)-1$ fids/B. The sextupoles are envisioned as $8 \mathrm{~cm}$ aperture, $16 \mathrm{~cm}$ effective length.

We have restricted the latice search to tunes which are far from integer and half-integer resonances and also the sum resonance $v_{x}+v_{y}=$ integer. We have particularly a:sided the integer "structure" resonances $v=4,8, \ldots$ In general. we constrained the solutions to tunes $\left(v_{x}, v_{y}\right)=(3.25 .3 .25)$, $(5.25,3.25),(5.25,5.25),(7.25,3.25)$ and $(7.25,5.25)$. We do not wish to imply from the above that the machine must operate at the linear coupling line $v_{x}-v_{y}=$ integer, but it should be capable of doing so if one desires $100 \%$ coupling. In Table (I) we summarize the properties of several interesting solutions. Quadrupole strengths are normalized, i.e. $g=G / B p$ with the gradient $G$ in $k G / m$ and $B \rho$ in $k G \mathrm{~m}$. The sign convention used here is that positive quadrupole strength means horizontally focussing. Since we have only three quadrupole strengths to vary, the programs were successful only when the number of constraints was 33 . we chose $v_{x}, v_{y}$ and $n_{x}^{*}=0$ where $\eta_{x}^{*}$ is the dispersion function in the long straight. With very strong wigglers, the beam emittance $\epsilon$ and hence size $\sigma=\sqrt{E B}$ will increase; an undesirable result for most storage ring applications. However, for the present application it would be desirable to increase the size. particularly at injection energy, and thereby increase the Touschek lifetime in Eq. (19). If this can be accomplished without a serious increase in the momentum compaction factor ap and thus a decrease in RF bucket size in Eq. (17) on which the bremsgtrahlung lifetime of Eqs. (9) and (16) depend, we may be able to avoid a boster synchrotron and inject directly into the storage ring at low energy. We have studied the case of $\pi_{x}^{*} \neq 0$ and will discuss the results later. For the present we are limiting the lattice search to the subset $\pi_{x}^{*}=0$. only two basic solutions were found with the conditions previously stated: $(3.25,3,25)$ and $(5.25,3.25)$. Solution $A$ of Table 1 is slight improvement over solution $B$ only with regard to less negative natural chromaticities $x$ and $y$ We must increase the chromaticity to positive values, as previously noted, with sextupoles of strength $s_{f}$ and $s_{d}$ in the $d_{j}$ straight section; the required strength is related to the chromaticity change $\Delta E$ byoj

$$
\begin{aligned}
& \Delta \xi_{x}=\xi_{x}-\xi_{x}^{0}=-\frac{N_{s}}{4 \pi}\left[N_{f} \beta_{x f} \eta_{x f} S_{f}+N_{d} \beta_{x d}{ }_{x d} S_{d}\right] \\
& \Delta \xi_{y}=\xi_{y}-\xi_{y}^{0}=\frac{N_{s}}{4 \pi}\left[N_{f} \beta_{y f} n_{x f} S_{f}+N_{d} \beta_{y d} n_{x d} S_{d}\right]
\end{aligned}
$$


where $N_{s}=$ number of superperiods

$N_{d}=$ number of "o" sextupoles per superpertad

$N_{f}=$ number of "fo" sextupoles per superperiod

$n_{x d}$ and $n_{x}$ are the horizontal eta functions at the indicated sextupoles, $B_{x f}, B_{x d}$ and $B_{y f}, B_{y d}$ are the horizontal and vertical beta functions at the indicated sextupole, $S_{f}$ and $S_{d}$ are the sextupole strengths $S_{d, f}=B^{\prime \prime} \mathrm{k} / B_{p} W i$ th 1 =effective length and $B=1 / 2 B^{\prime \prime} x^{2}$ the sextupole field in the median plane. Eq. (21) can be solved for $S_{f}$ and $s_{d}$ to obtain

$$
\begin{aligned}
& s_{f}=\left(4 \pi \Delta \xi_{y} \beta_{x d}+\Delta \xi_{x} \beta_{y d}\right) / N_{s} N_{f} \eta_{x f}\left(\beta_{x d} \beta_{y f}-\beta_{x f} \beta_{y d}\right) \\
& s_{d}=-4 \pi\left(\Delta \xi_{x} \beta_{y f}+\Delta \xi_{y} \beta_{x f}\right) / N_{s} N_{d} \eta_{x d}\left(\beta_{x d} \beta_{y f}-\beta_{x f} \beta_{y d}\right)
\end{aligned}
$$

It is well known from particle tracking calculations" that introduction af nonlinear elements such as sextupoles into the lattice will decrease the dynamic aperture of the machine-- the aperture required to contain electrons which acquire large amplitude betatron oscillations following photon emission. With sufficient sextupole strength it is indeed possible to increase the growth rate of the oscillation amplitude to values greater than the radiation damping rate, in which case the solution becomes unstable. So we will be looking for solutions with small co. $^{\circ}$.

Another consideration is the kicker strength required for the injection "bump". We desire a local orbit deformation of the circulating beam the injection septum magnet in order to minimize the oscillation amplitude of the injected beam relative to the bedil. i-eady stored in the machine. This condition is most easily achieved with. "bump" which extends for one-half of a betatron wavelength $\lambda_{p}$ centered about the injection septum. A perfect orbit deformation would then consist of two "kicker" magnets at $\lambda_{a} / 4$ on ither sita of the septum (a petatron phase shift of $\downarrow=0.25 \times 2 \pi$ radians). However. it is seldom possible to attain this condition and a thirdkicker is usually required3s preferably near the septum, to obtain a local deformation. We have allowed space in the upstream end of the injection straight for this correction kicker. In any case, the amplitude $k$ of the "kick" at point $k$ in the d straight is related to the amplitude $d$ of the deformation at the center of the long straight (approximately the septum position) by

$$
d=\sqrt{B_{x K^{2}}{ }^{\star}} \sin \psi_{K}
$$

A) though the $\psi_{k} / 2 \pi=0.19$ of solution $A$ is closer to the optimum value of 0.25 , the values of $B_{x k}=1.5 \mathrm{~m}$ and $B_{x}^{*}=10.12 \mathrm{~m}$ of solution B are sufficient!y larger than in solution A to yiold a smaller kick requirement k 
for a given deformation in Eq. (23). For a typical value $d=4 \mathrm{~cm}$ the required kicks would be $k_{z}=16.7 \mathrm{mrad}$ and $k_{b}=12.2 \mathrm{mrad}$. These values are readily attainable with a full aperture (window-frame) ferrite kicker outside of a ceramic vacuum chamber. For example, consider a kicker magnet of $8 \mathrm{~cm}$ aperture with a field of $2.5 \mathrm{~kg}$ which is attainable with good quality ferrite. ${ }^{36}$ If the magnet effective length is $l=0.5 \mathrm{~m}$ and the magnetic rigidity at $E_{0}=0.1 \mathrm{GeV}$ injection energy is $46.7 / 14=3.335 \mathrm{~kg} \mathrm{~m}$ we obtain a peak deflection of $k_{\max }=375 \mathrm{mrad}$. Even if we inject at full energy the kicker capability is $k_{\max }=26.8 \mathrm{mrad}$. We can therefore tolerate much smaller values of $\beta_{x k}, B_{x}^{*}$ and $\sin \phi_{k}$ than in either solutions $A$ or $B$.

For solution $B$ we also give the values of several key parameters at a tentative injection energy of $0.1 \mathrm{GeV} ; \tau_{x}=9.73 \mathrm{sec}, \sigma_{x}^{\star}=0.13 \mathrm{~mm}$ and $\sigma_{a}=5 k e v$ These values are of course completely unreasonable for injection into the storage ring. In Fig. (5) we show the energy dependence of the parameters and note that they are changing rapidly at low energy. Solutions $c$ and $D$ show the effect of the wiggler on the damping time $\tau_{x}$, beam sizes $\sigma_{x}^{*}$ and $\sigma_{z}$ and energy spread $\sigma_{0}$. The decrease in $r_{x}$ at $0.1 \mathrm{GeV}$ to 0.6 sec when the wigglers are turned up to twice their nominal value $(2 \times 80 / 14=11.4 \mathrm{kG})$ is a significant improvement. Note that the computer code assumes a symetric machine with reflectional symmetry about the center of QF 2 . Hence, it assumes that four wigglers of the indicated strength are present. If we have only one wiggler, it would be at feur times the quoted strength. Note also that, in solution 0 , the value of $\beta_{y}^{*}$ has decreased to $0.05 \mathrm{~m}$ as a result of the strong vertical fringe field focussing introduced by the wiggler (and accompanying vertical tunn shift $\Delta \nu_{y}$ which accounts for the reduction in $Q D_{1}$ strength from solutions $B$ to 0 ). At slightly over $2 x$ nominal strength. $B_{y} \rightarrow 0$ and the vertical tune $y_{y}=1,2 \pi f d s / B_{y}$ diverges at which point no stable solutions exist. The energy dependence of the injection parameters with the wigglers on is shown in Fig. (6) and exhibit the same rapid change in parameters at low energy noted in Fig. (5).

For the $v_{x}, v_{y}=(5.25,3.25)$ solutions $E, F$ and $G$, there appears to be no advantage relative to the lower tune solutions. The more favorable phase shift $\phi_{k} / 2 \pi=0.27$ does not compensate for the much smaller value of $\sqrt{\beta_{x k} \beta_{x}^{*}}$ in Eq. (23) and the natural chromaticities $\xi_{x}^{\circ}, \xi_{y}^{\circ}=-10.22,-24.75$ are too negative. Also, the peak value $\hat{\beta}_{y}=66.64 \mathrm{~m}$ would give a significant decrease in the coulomb scattering lifetime in Eq. (13). Therefore, the best $L=51 \mathrm{~m}$ ring we found is solution $B$. We are not satisfied with the large value of $a_{p}=0.087$, however. The NSLS VUV ring ${ }^{33}$ for example, has $a_{p}=0.023$. One final comnent an rable 1 . The largest quadrupole strength encountered is $4.57 \mathrm{~m}^{-2}$. At $B_{p}=46.69 \mathrm{kG} \mathrm{m}$ this corresponds to a gradient of $2.134 \mathrm{kG}^{-1}$. If we intend to use quadrupoles of $4 \mathrm{~cm}$ half aperture, then the pole-tip field is $8.53 \mathrm{kG}$. For the NSLS X-ray ring ${ }^{3}$ the peak field specified is $5 \mathrm{kG}$ and will increase to $6 \mathrm{~kg}$ for $3 \mathrm{GeV}$ operation. For the proposed 38 low emittance SPEAR configuration at $3 \mathrm{GeV}$, the maximum gradient will be $0.84 \mathrm{~kg} / \mathrm{m}$ and the corresponding pole-trip field $B_{t}=6.3 \mathrm{kG}$. It is expected 39 that the SPEAR quadrupoles have this capability based on previous experience with 4 GeV SPEAR operation. We are, however, again encouraged by the successful operation of the SLAC damping ring" using $B_{t}=8.06 \mathrm{kG}$ quadruples and are making the bold extrapolation to Bt $\leq 10 \mathrm{~kg}$.

B. Lattices with $15^{\circ}$ dipoles, $L=53.96 \mathrm{~m}, \mathrm{~h}=90$ 
The $h=90$ lattice is a 4 quadrupole (per half superperiod) variant of the A lattice. Its geometry is given in the sketch below.

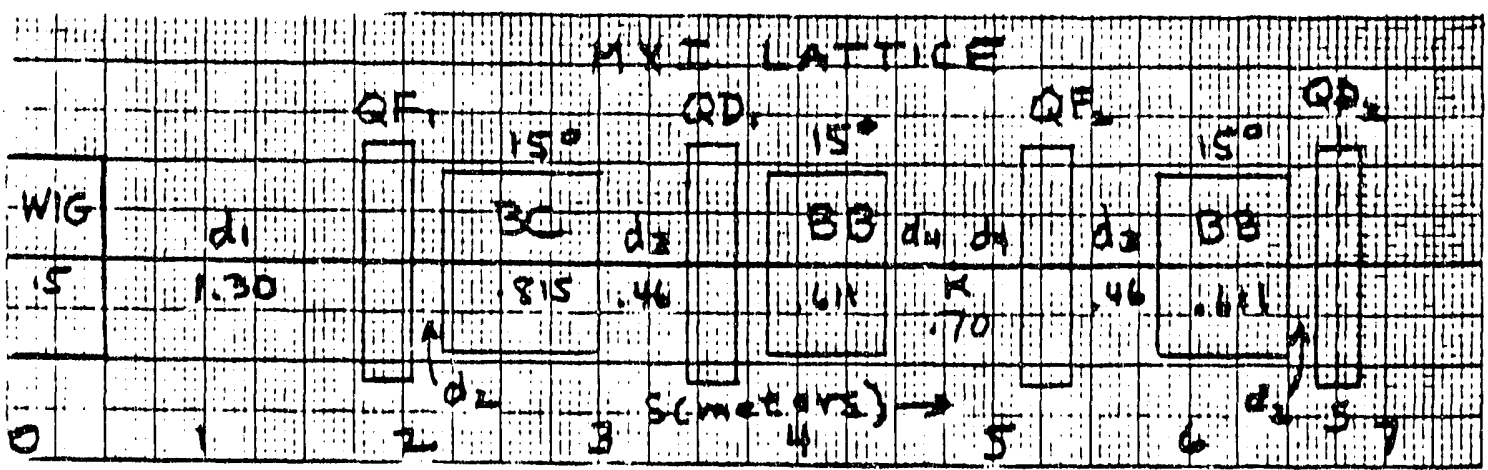

The addition of an extra quadrupole could be an important aid in finding new solutions; however, we again limited the search to 3 constraints; $\nu_{x}, \nu_{y}$ and $n_{x}^{*}$. Some of these solutions are presented in Table 11. We first note that the computer program desires a horizontally focussing quadrupole for $\mathrm{QO}_{2}$ in spite of an initial negative guess value and numerous attemptis to find a solution with vertically focussing $Q_{2} \mathrm{O}_{2}$. Thus, solutions $A$, B arid $C$ are, in a sense, equivalent to the $L=51 \mathrm{~m}$ ring as is evident from comparison of I $B$ and II $A$. However, the kicker parameters are nearly optimal with $\phi_{k} / 2 \pi=0.27, \beta_{x k}=1.53 \mathrm{~m}$ and $\beta_{x}^{*}=11.59 \mathrm{~m}$. The momentum compaction factor $a_{p}=0.072$ is somewhat smaller than solution 1.B. The required quatrupole strengths are smaller, which is helpful. The parameter which argues most strongly in favor of solution II A is the maximum value of the eta function. $\hat{n}_{x}=1.35 \mathrm{~m}$, which is a factor of more than two smaller than for the $I$ ring. since the equilibrium orbit shift of an off-momentum particle is

$$
\left(\Delta_{\mathrm{X}}\right)_{\mathrm{E} .0 .}=\pi_{\mathrm{x}}\left(\Delta \mathrm{p} / \mathrm{p}_{\mathrm{o}}\right)
$$

ring II $A$ is capable of twice the momentum acceptance of $r i n g$ I $B$ if one is able to increase the RF bucket size of $E q$. (17) with a sufficiently large peak RF voltage Vm.

In solutions II $B$ and II $C$ we give the results for the same ring with the wigglers on at normal and $2 x$ normal values, respectively. The results are similar to Table I $C$ and I $D$ solutions. We did not compute the E. $=0.1 \mathrm{GeV}$ case for II $A$ and II $B$. It should also be pointed out that all solutions in this report were calculated with a peak $R F$ voltage of $V / m=2.4$ MV and thus the energy lass of $2.166 \mathrm{MeV} /$ turn for solution II $C$ at $E_{0}=1.4$ GeV gives values for parameters dependent on the RF bucket size (and phase-stable angle $\phi_{s}=115.5^{\circ}$ in II C) such as $\sigma_{z}$ and $\sigma_{e}$ which are smaller than in a realistic machine design with adequate RF overvoltage. For example, solution II C gives $2 \times 10^{-6}$ min for the quantum lifetime $T q$ whereas the value of that parameter is essentially infinite $\left(2 \times 10^{3} 1\right.$ min in solution $\left.11 \mathrm{~A}\right)$ when the overvoltage Vm is generous. Solution II $A$ is the best solution we have found in the present 
study and we therefore present the computer input for the case in Table II!, the computed betatron functions in Table IV and the resulting parameters in Table $V$. We have also studied the effect of non-zero $n_{x}^{*}$ with the wiggler on at $2 \times$ nominal strength at a possible injection energy of 100 MeV to observe the beam blow-up effects associated with non-zero momentum dispersion in a region of large electron energy changes due to radiation. (The Rf cavity is another region of large electron energy changes.) The results for beam sizes $\sigma_{x}^{*}, \sigma_{y}^{*}$ (with $100 \%$ coupling assumed) and $\sigma_{z}$ and the momentum compaction factor $\sigma_{p}$ are given in Fig. (7). In the interval $0 \leq \pi_{x}^{*} s 1.5 \mathrm{~m}$ the values $\sigma_{x}^{*}$ and $\sigma_{y}^{*}$ inorease by a faotor of $\leq 3$. The increase in $\sigma_{z}$ is smaller but we distrust this result for the reasons previousiy discussed. A small inarease in $a_{p}$ results. These changes are accomplished by a relatively small change in quadrupole strengths and we could presumably realize much larger changes in beam size. Thus, we can realize at least an order of magnitude increase in Touschek lifetime of $E q$. (19) at injection energy. The calculation does not indicate an increase in energy spread $\sigma E / E$ vs $n_{x}^{\star}$ so presumably the integral of Eq. (19) remains constant.

one remark on the wiggler bend angles stated in Tables I and II. In the sketch below we show one half of the assumed wiggler geometry with reflectional symmetry. A full pole has an effective length of $8 \mathrm{~cm}$ with drift spaces of $2 \mathrm{~cm}$ between poles. The total halp length of the wiggler is $50 \mathrm{~cm}$ and includes a half pole BWPH and BWMH at either end. With magnetic rigidity $B \rho=46.69897333 \mathrm{~kg} \mathrm{~m}$ and $a$ field of $80 \mathrm{kG}$. the bend radius is $\rho=0.583737167 \mathrm{~m}$. The bend angle of a full poie is then $\theta=0.137047981$ $\mathrm{rad}=7.85227090^{\circ}$.

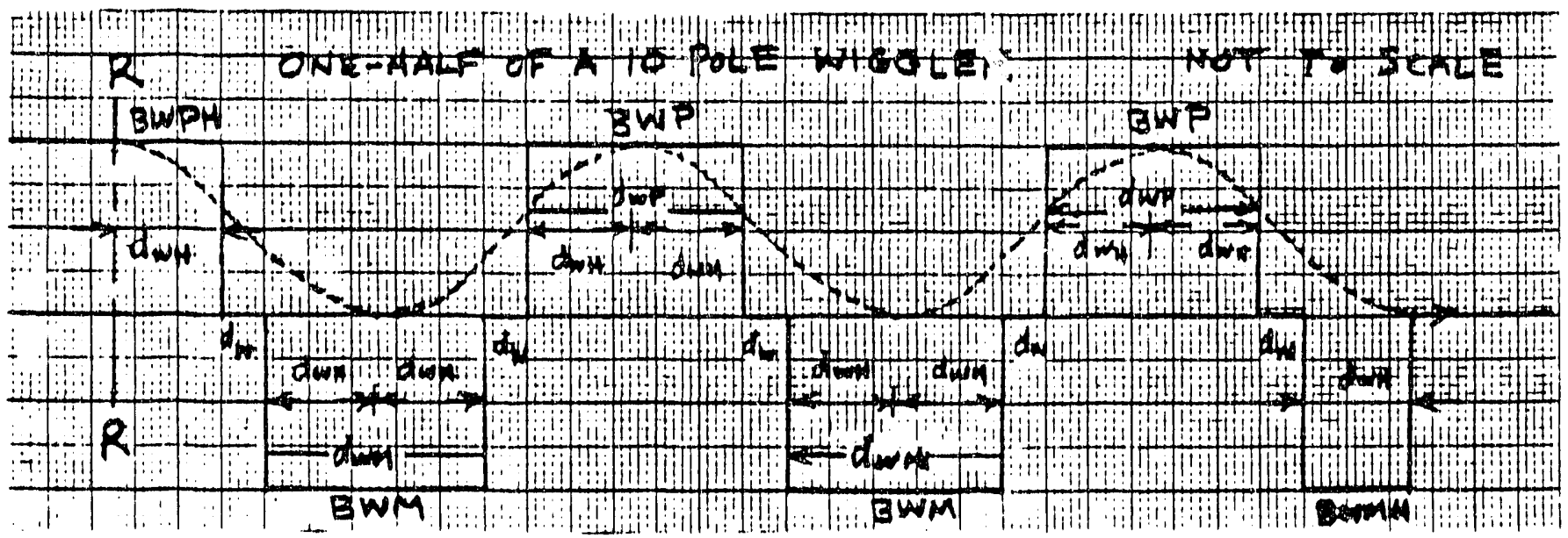

For the $L=53.96 \mathrm{~m}, h=90,15^{\circ}$ dipole ring we also looked for solutions at $\nu_{x}, v_{y}=(5.25,3.25)$ with the expectation that a higher horizontal tune would yield a smaller dispersion function $n_{x}$ and smaller momentum compaction factor $a_{p}$ than solution II $A$. It was first apparent that, with larger $v_{x}$ the tune shift from the kicker to the injection septum (center of the long straight) was much too large. We therefore did the calculations with the kicker straight section $d_{4}-d_{4}$ located immediately downstream of the BC magnet (interchange of $d_{3}$ with $d_{4}-d_{4}$ in sketch of MXI lattice). This resulted in a 
nearly optimum phase of $\phi_{k}=0.27 \times 2 \pi$. The $a_{p}$ and $\hat{\eta}_{x}$ values do decrease slightly but two parameters change in a detrimental manner; the value of $B_{x}^{*}$ decreases from $11.59 \mathrm{~m}$ in II A to $0.29 \mathrm{~m}$ in II D whioh implies that the injection kicker would require a $k=55.2 \mathrm{mr}$ deflection to give a $4 \mathrm{~cm}$ displacement at the septum, a value which exceeds the kicker capability if we elect to inject at full $1.4 \mathrm{GeV}$ energy. Further, the maximum vertical $B$ value $\hat{B}_{y}$ has increased to $47.7 \mathrm{~m}$ from $29.1 \mathrm{~m}$ which will decrease the gas scattering lifetime of Eq. (13). We attempted increasing the value of $B_{x}^{*}$ by imposing a constraint in case II $G$ but the value of $\hat{\beta}_{y}$ increased still further to $53.80 \mathrm{~m}$ and the kicker phase increased to $\psi_{k}=0.40 \times 2 \pi$ as a result. The value of $\psi_{k}$ is unacceptabiy large. Since we have 4 variable quadrupoles, we tried imposing 4 constraints; on $B_{x}^{*}=0.5 \mathrm{~m}$ and $n_{x}^{*}=-0.5$ in addition to $\nu_{x}$ and $\nu_{y}$ but this result (not shown) increased $\hat{B}_{y}$ to $119 \mathrm{~m}, a_{p}$ to 0.135 , and natural chromaticity $\xi_{y}^{0}$ to -25.3 ; all unacceptably large. Hence solution II $A$ is the only $L=53.96 \mathrm{~m}$ solution which meets our requirements.

c. Lattices with $11.25^{\circ}$ dipoles, $L=58.76, h=98$

The $h=98$ lattice has 5 quadrupoles and 4 dipoles per half-superperiod as shown in the sketch below. Again, the addition of one additional quadrupole gives the machine designer another degree of freedom in choosing constraints.

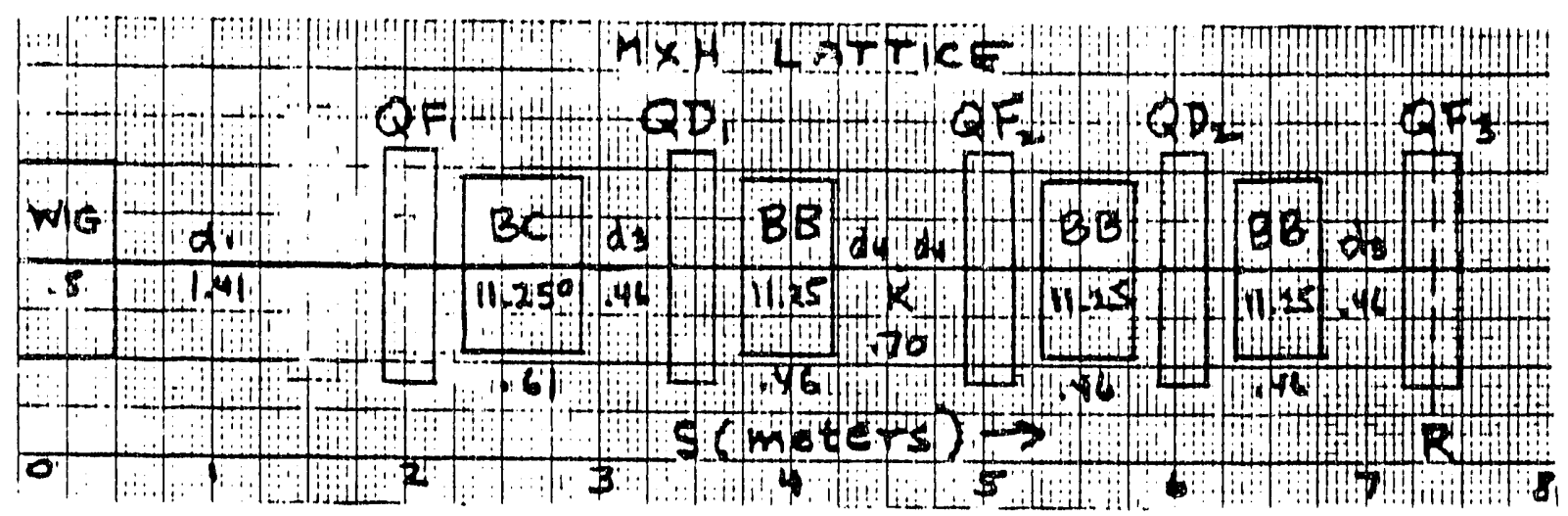

We have generally used 4 constraints $\nu_{x}, \nu_{y}, n_{x}^{*}=0$ and $\beta_{y}^{*}=0.5 \mathrm{~m}$ and we have searched for solutions with $\nu_{x} \nu_{y}:(3.25,3.25),(5.25 .3 .25),(5.25,5.25)$ and $(7.25 .3 .25)$. The solution at $\nu_{x}, \nu_{y}=3.25,3.25$ (VI A) is acceptable in all respects. The most significant improvement relative to the results of Table II is the smaller eta function $\hat{\pi}_{x}=1.90$ and momentum cumpaction factor $a_{p}=0.053$. The injection situation is quite favorable even with a somewhat small kicker phase $\psi_{x}=0.16 \times 2 \pi$ because the beta functions $\beta_{x}^{*}=20.04 \mathrm{~m}$ and $\beta_{x k}=15.26 \mathrm{~m}$ are so large. The results of solution VI $\theta$ with the wigglers on are equally acceptable. For solution VI $c$ at $v_{x}, v_{y}=5.25,3.25$ we have relaxed the constraint $\beta_{y}^{*}$ from the optimum $0.5 \mathrm{~m}$ to $1.0 \mathrm{~m}$ to avoid a solution (5/24-7, not shown) for which the $B_{y}^{*}=0.5 \mathrm{~m}$ constraint resulted in an unacceptably large peak value $\hat{\beta}_{y}=62.57 \mathrm{~m}$. The excessively large kicker phase $\psi_{k}=0.43 \times 2 \pi$ somewhat improved by relocating the kicker in solution 
VI o downstream of the QD, quadrupole and could no doubt be improved still more by further movement of the kicker upstream. The solutions $V I C$ and $D$ are particularly attractive because of the very small value of the eta function $\hat{n}_{x}=0.90 \mathrm{~m}$, but this advantage is sumewhat offset by a decrease in chromaticity $\xi_{y}^{0}=-16.15$. We feel that $V I D$ is a usable solution. For the $\nu_{x}, \nu_{y}=5.25,5.25$ case we found only one solution, $V I E$, and this result appears unacoeptable to us because of the kicker parameters $\phi_{k}=0.47 \times 2 \pi, \beta_{x k}=0.58 \mathrm{~m}$ and $B_{x}^{*}=0.12 \mathrm{~m}$. A.1so, $a_{p}=0.144$ is much too large. The maximum quadrupole strength of $5.17 \mathrm{~m}^{-2}$ would result in a pole tip field of $B_{t}=9.65 \mathrm{kG}$ which is high but within our previously stated limit of $\leq 10 \mathrm{kG}$. No doubt the injection situation could be improved at this tune by moving the kicker upstream (it was back in the original location given on the $M \times H$ lattice sketch for VI E) but this case was not pursued in the present study.

Finally we looked briefly at the $\nu_{x}, v_{y}=7.25,3.25$ possibility. In VI F the search was constrained to only $\dot{v}_{x}$ and $v_{y}$. The resulting kicker phase $\psi_{k}=0.68 \times 2 \pi$ is, of course, impossible but for this run the kicker position was as given in the MXH lattice sketch; just upstream of $Q F 2$. We, therefore, moved it as far upstream as possible--between the BC dipole and QD, quadrupole for solution VI $G$. Constraints $B_{y}^{*}=1.0 \mathrm{~m}$ and $n_{x}^{*}=0$ were also imposed. The resulting $\psi_{k}=0.46 \times 2 \pi$ is still unacoeptably large and $B_{x k}=0.17 \mathrm{~m}$ and $B_{x}^{*}=0.5 \mathrm{~m}$ are too small for efficient injection. Also, the uncorrected chromaticities $\xi_{x}=-24.14$ and $\xi_{y}=-34.53$ are too negative and the peak vertical beta function $\hat{B}_{y}=72.32 \mathrm{~m}$ is too large. We, therefore, rule out this solution.

II. Discussion

We will restrict this discussion to evaluation of the required aperture of the machine, the coulomb scattering and breinsstrahiung lifetimes of the Table II A 5/29-15 53.96 $\mathrm{m}$ MXI ring and the beam current limit for an adequate Touschek lifetime at injection and operating energy. The $53.96 \mathrm{~m}$ ring is our preferred choice of machine from among the (large) subset of possible designs because it is less costly than the MXH $58.76 \mathrm{~m}$ design but technically better in several respects than the $50.96 \mathrm{~m}$ ring. Choice number 2 is the Table VI A MXH 5/24-1 $58.76 \mathrm{~m}$ ring which provides the designer with smaller $n_{x}$ and $a_{p}$ values and considerably more flexibility in optimization of the lattice. Perhaps most important is that the MXH design uses $11.25^{\circ}$ dipoles and a sagetta of $S \approx 1 \mathrm{~cm}$; we are uncomfortable with the $S=2 \mathrm{~cm}$ of the $15^{\circ}$ dipole rings. Choice number 3 is the Table I A $50.96 \mathrm{~m} \mathrm{MXJ}$ design $5 / 22-7$ in Table I A because it is the least expensive and smallest and appears to satisfy the requirements; however the larger $\hat{\pi}_{x}$ and $a_{p}$ of that machine are points of discomfort. We will not know the full significance of these factors, as well as the affect of nonlinearities such as the chromaticity-correcting sextupoles, without tracking calculations which evaluate the dunamic aperture of the machine with nonlinearities but are at present beyond the scope of this report. our goal has been to find a suitable linear lattice configuration. It is perhaps useful to note that we have been unable to find a configuration with $a_{p}$ as low as the present SPEAR value of 0.0417 or the projected value of 0.0166 for the low emittance SPEAR configuration 38 or the $\alpha_{p}=0.0234$ of the NSLS VUV ringjo; this problem appears intrinsic to our basic geometric choice of quadrupole singlets in the long straights. 
We first require the RF bucket size of MXI 5/29-16 from Eq. (17) with $V_{m}=2.4 \mathrm{MV}, E_{e}=1.4 \mathrm{GeV}, h=90$ and $a_{p}=0.072$. The RF bucket from these parameters is given in Fig. (8) and we coinpute $\Delta E_{r f}=18.18 \mathrm{MeV}$ from Eq. (17); thus $\pm \Delta E_{r} f / E_{s}= \pm 1.30 \%$ (The computer result from Fig. ( 8 ) is $17.38 \mathrm{MeV}$, probably due to inacouraoy in single precision arithmetic on the VAX.) Here is where we would have profited from a factor of four smaller $a_{p}$ since larger $V m$ is hard to obtain. The peak value $\hat{\eta}_{x}$ is at $Q f_{z}$ from table IV, at which point we can accommodate equilibrium orbits with a momentum spread

$$
\left(\Delta_{t} / p_{0}\right)_{\max }=\left(\Delta E / E_{0}\right)_{\max }{ }^{m a / \hat{n}_{x}}
$$

We can then acoommodate $\pm 2.97 \%$ spread in equilibrium orbits in this machine in the "zero beam size" limit. The amount of aperture actually required about the equilibrium orbit call be determined accurately only by traoking calculations with a realistic model of the machine which would include higher order multipole content in the dipoles (mostly sextupole) and quadrupoles. We can. however, estimate the aperture requirement by first calculating the emmittance $\varepsilon_{x}$ of the damped beam from the definition $\sigma=r E^{2}$ and the values $\sigma_{x}^{*}$ of the damped beam size and $B_{x}^{*}$ irl rable IV. We will be conservative here and use the value $\sigma_{x_{0}^{*}}^{*}=2.633 \mathrm{~mm}$ (no coupling-the values of $\sigma_{r}^{*}$ in Tables I, II and $V I$ are for $100 \%$ coupling) froin Table $V$. We then obtain for the damped emittance $\varepsilon_{x_{0}}=0.5981 \mathrm{~mm} \mathrm{mr}--a$ ather large damped emittance for present day electron storage rings. For colnparison we normalize $\varepsilon_{x o}$ by dividing it by $E_{0}^{2}$ since this is the energy dependence of the emittance; then $\left(\varepsilon_{\times 0}\right)_{n}=0.305 \mathrm{~mm} \mathrm{mr} G \mathrm{eV}^{-2}$ whereas the present SPEAR normalized emittance is

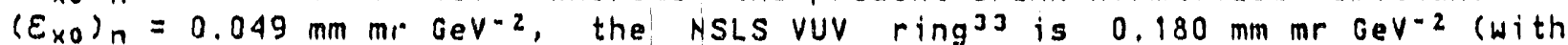
solutions down to $\left.0.041 \mathrm{~mm} m \cdot G e V^{-2}\right)$, the new low-emittancejo SPEAR configuration is $0.014 \mathrm{~mm} \mathrm{mr} G e V{ }^{2}$, the NSLS $x-r$ ay ring $^{37}$ is $0.013 \mathrm{~mm} \mathrm{mr} \mathrm{GeV-2}$ and the proplosed very low emittance ALS ring for the Berkeley NCAM project"o is $0.003 \mathrm{~mm} \mathrm{mr} \mathrm{GeV-2}$. Thus, the $\mathrm{MXH} 5 / 29-16$ configuration is a high emittance one; fortunately we do not require a low emittance configuration for the present application. In the absence of tracking, we can use a "rule of thumb" estimate" "which we used initial y for the dynamic aperture Ad requirenent for the NSLS:

$$
\begin{aligned}
& A_{D H}=100_{x}+10 \mathrm{~mm} \text { (horizontal) } \\
& A_{D V}=100_{y}+; \mathrm{mm} \text { (vertical) }
\end{aligned}
$$

The largest $B_{x}$ value from Table IV is at $Q F$, with $\hat{\beta}_{x}=11.869 \mathrm{~m}$. With the $\varepsilon_{x 0}=0.5981 \mathrm{~mm} \mathrm{mr}$ we get $\left(\sigma_{x}\right)$ max $=2.664 \mathrm{~mm}$ and $A_{\mathrm{dm}}=36.64 \mathrm{~mm}$. Since $n_{x}=0$ at $Q F$, from Table IV we require no additional aperture at this point for off-set of the equilibrium ortit $\eta_{x}(\Delta p / p o)$ due to momentum off-set. So our prior use of $4 \mathrm{~cm}$ for the half aperture of the quadrupoles appears to be a good choice. Note in Eq. (25) that the addition of $10 \mathrm{~mm}$ to $10 \sigma_{x}$ is an 
estimate of the additional aperture required by olosed orbit error and betatron oscillation amplitude of the initially injeoted beam before it damps down to the values used above.

We can now estimate the coulamb scattering lifetime from Eq. (13). It is generally the case in storage rings that the physical aperture is smallest in the vertical plane because $i t$ is the vertical gap of bending magnets and horizontally deflecting wigglers that one wishes to minimize for reason of cost. The maximum vertioal $\hat{B}_{y}=29.14 \mathrm{~m}$ is at quadrupole $Q D_{1}$. For the purpose of this caloulation we wlll assume that the vacuum chamber is a rectangular, thin-walled stainless steel chamber which has been used sucoessfully at Daresbury rather than the thioker walled (4 mm) extruded aluminum chamber used elsewhere; thus, the inside dimensions can be approximately $8 \mathrm{~cm}$ horizontal and $4 \mathrm{~cm}$ vertioal. We further assume that nearly all of the residual gas is hydrogen: this conclusion for a wellconditioned ultra-high vacuum system after baking and desorption of gas from the walls is substantiated by results of vacuum experts at several laboratories.4 204445 Thus $z=10$ For $\left\langle\beta_{v}\right\rangle$ we use the weighted average of $9.5 \mathrm{~m}$. The final quantity required is the particle density of soattering nuclei $n$. An estimate of n requires knowledge rot only of the static pressure $\left(\approx 10^{-11}\right.$ Torr) but also the desorption rate of molecules from the chamber wall due to synchrotron radiation which is a funotion of beam current, and finally the density of trapped ions oreated by the beam. This is a difficult quantity to estimate with confidence. I, therefore, will soale from knowledge" 6 of the $24 \mathrm{hr}$ beam lifetime of SPEAR at 3 GeV when the machine is well conditioned and the vertical aperture at the $Q D$ oell quadrupoles where the vertical half-size of the ohamber is $22 \mathrm{~mm}$ and $B_{y}=20.6 \mathrm{~m}$. For $\langle\beta v\rangle$ for SPEAR we use $12.5 \mathrm{~m}$. Then Eq. (13) gives $n=5.0410^{10}$ nuolei $\mathrm{cm}^{-3}$ which corresponds to an impressively low value of $p=6.3610 .11$ Torr. If the observed lifetime is not limited by gas soattering. then this is an upper Iimit on the SPEAR pressure. For a higher current machine with smaller spacing between bunches (the effective distance between the "four" SPEAR bunahes is $58.5 \mathrm{~m}$ ) the ion density will be larger so we will use 10.10 Torr $\left(n \approx B \quad 1010 / \mathrm{cm}^{3}\right)$. The gas scattering lifatime at 1.4 GeV for our machine is then

$$
r_{0}=9110 \mathrm{sec}
$$

At $0.1 \mathrm{GeV}$ we scale by $\gamma^{2}$ and have $r_{0}(.1 \mathrm{GeV})=46.5 \mathrm{sec}$. Thus, $\tau_{0}$ does not rule out $100 \mathrm{MeV}$ injection if the injector is fast-pulsing. The pulse rate of the injector, however, is limited by the damping time which we have seen, in Table I, can be reduced with use of wigglers to 0.6 sec. Hence gas scattering is not the limiting factor for low energy injection.

One final point on scattering. We have tried throughout this study to keep the value of $\beta_{y}^{*}$ at the wiggler small and, where possible, near the optimum value of $0.5 \mathrm{~m}$ to obtain the smallest vertical beam through a $1 \mathrm{~m}$ long wiggler. In solution II $A \quad 5 / 29-16$ we came close with $B_{y}=0.79 \mathrm{~m}$ without constraint on that parameter. The coupled vertical emittance from table IV is then

$$
\varepsilon_{y(A)}=\left(\sigma_{y(A)}^{*}\right) \beta_{y}^{2 / *}-301 m m r
$$


and the vertioal beam width (standard daviation) at the end of the wiggler is. from Eq. (7), $\sigma_{y}=\sqrt{\varepsilon_{y}(1) \bar{B}}=0.310 \mathrm{~mm}$. Then the aperture requitrement from Eq. (25) is $\pm A d v= \pm 8.1 \mathrm{~mm}$. The present $60 \mathrm{kO}$ NSLS wiggler has a vertioal aperture of $\pm 9 \mathrm{~mm}$ and is thus adequate for this machine at full ellergy. At injeotion energy the damped vertioal beam size will be oven smaller since - $\alpha E^{2}$ and the problem we will enoounter is the emittance of the injected beam. If we use the typical value of $1 \mathrm{~mm} \mathrm{mr}$ whioh pertains the Wisconsin 100 MeV miarotron, the o y of the injeoted beam inoreases by a factor of 1.796 to $\sigma_{y}=0.557 \mathrm{~mm}$ and the aperture requirement beoomes $A_{d v}= \pm 10.57 \mathrm{~mm}$ at the wiggler. This problem mav constitute an argument for a Booster sunchrotron injeotor in which the beam from the pre-injeotor will damp to values comparable to those of the storage ring oited above, partioularly if the pre-injector is a $100 \mathrm{MeV}$ linac with a larye emittance - $=E / \pi=6.37 \mathrm{~mm} \mathrm{mr}$ which pertains 4 o for the NSLS 100 MeV linao pre-injeotor. The alternative to the wiggler as an injection aperture is to build a variable gap wiggler or one that moves out of the maohine aperture at injeotion. It seems to us that this is a formidable meohanical problem for a superoonducting devioe of the type contemplated here and we rejeot that method as a viable al ternative.

The next parameter to be evaluted is the bremasstrahlung lifetime of Eq. (9) with the oross-section of Eq. (16). The dependence of the orossseotion on electron energy is weak; thus, if the lifetime is large enough at operating energy, it should suffice at injeotion energy. We use the Rf bucket size of $\Delta E_{r+}=18.18$ MeV calculated earlier in this chipter and the particle density of $n=8 \times 10^{10} \mathrm{~cm}^{-J}$ used in the estimate of gas soatteringlifutime. Eq. (16) gives at $=1.4 \mathrm{GeV} \sigma_{b}=59.582 \mathrm{millibarns}$ and Eq. ( 9 ) gives the lifetime

$$
r_{b}=6993 \mathrm{sec}
$$

If we assume that the same RF peak voltage is maintained at 100 MeV injection energy, then the $R F$ bucket size at that energy is, from Eq. (17), $\Delta E_{r f}=4.858 \mathrm{MeV}$, the bremsstrahlung ross-section is ob $=38.445 \mathrm{mb}$ and $T_{b}=10838 \mathrm{sec}$.

Finally, we evaluate the Touschek lifetime of Eq. (19), first it 1.4 GeV and then at the proposed 0.1 GeV injection energy. The maximum momentum acceptance $\Delta p_{m}$ in units of $m_{0} c$ is, at relativistic energy, numerically equal to the maximum $R F$ bucket acceptance $\Delta E_{r f}$ divided by moc ${ }^{2} i . e$. $\Delta p_{m}=18.18 / 0.511=35.577$. The integral is given in terms of the parameter $n=\Delta p \pi_{0}=0.013$ at $\gamma_{a}=2739.7$. Similarly, the parameter op is, relatavistically, equal to the energy width of the beam whioh from Table II is $\sigma_{0}=7.610 \%(1400)=1.064 \mathrm{MeV}$. In units of moc, $a_{p}$ is the dimensioniess number $\Delta \gamma=\gamma\left(E_{0}+\sigma_{a}\right)-\gamma\left(E_{0}\right)=2.0822$. From Fig. (4) we then get $9 \approx 0.805$. Then, from rable $V$ we get the values $\sigma_{x}^{*}(w)=0.186 \mathrm{~cm}, \sigma_{y}^{*}(a)=0.049 \mathrm{~cm}$ and $\sigma_{z}=1.119 \mathrm{~cm}$. The subscript A means the value with $100 \%$ coupling which we have been using (the zero coupling value of $\sigma_{y}$ is zero). The number of particles per bunoh $N_{0}$ is related to the beam current I and Rf frequency frf by $I$ (AMPS) $=$ eNof $N_{\text {of }}$ with $e=1.60210^{-19}$ coulombsrelectron. Note that $N_{0}$ is then proportional to $1 / f r f$ and thus the Touschek lifetime is proportional to frf. Substituting $1=0.4$ Amp, our desired ourrent, and the $f_{r f}=500 \mathrm{Mhz}$ which was chasen for this study (fallowing Daresbury. The DESY storage rings OnRIS and PETRA and the proposed LBL ALS ring), we have $N_{0}=4.994 \times 10^{9}$ el atrons/bunch. The Tousohek lifetime is then 
of greater interest is the lifetime at $100 \mathrm{MeV}$. We oan soale Eq. (28) by $\mathrm{r}^{\mathrm{s}}$ and obtain $\tau_{t o u} \approx 5 \mathrm{seo}$. Done more carefully we first note that the energy buoket oannot realistioally be inoreased to as large as the spread $\Delta p / p o$ of equillbrium orbits of $\pm 3 \%$ previously estimated from $\eta_{x}$. If we maintain $V_{m}$ at $2.4 \mathrm{MV}$ we would get $4.8 \mathrm{MeV}$ whluh is too large. So we arbitrarlly set the RF buoket $\Delta E_{p t}=2 \mathrm{MeV}(2 \%)_{*}$ This gives $\Delta p_{m}=2 / 0.511=3.914$. Next, the values of $\sigma_{x(*)}^{*}=0.0122 \mathrm{om}_{*} \sigma_{y(1)}^{*}=6.010^{-4} \mathrm{am}$ and $\sigma_{z}=0.0589 \mathrm{om}$ can be obtained from Fig. (7) for $\eta_{x}^{*}=0$. Then the parameters of the Touschek integral are $n=\Delta E_{r}+\gamma_{0}=0.02$ and $\sigma_{p}$ is obtained from the Table II $c$ value of $\sigma_{0}=1.510^{-4} \times 100=0.015 \mathrm{MeV}$ and the result $\sigma_{p}=\Delta y=\gamma(100.015)-\gamma(100)=0.029$. This value of $\sigma_{p}$ is too sinall to obtain from the $n=0.02$ ourve of Fig. (4) but we estimate $\mathcal{Q} \approx 3$. Then, with the same value of $\mathrm{Na}_{a}$ for $!=400 \mathrm{~mA}$ Eq (19) gives

$$
r_{\text {tou }}=0.37 \text { seo at } 0.1 \text { gev }
$$

We require an increase in $r$ tou of at least $10^{2}$ to even contemplate this injection energy. We have seen from $F i g$. (7) that simply changing the $n_{x}^{\star}$ value with the wigglers on oan inorease $\sigma_{x}^{*}$ and $\sigma_{y}^{*}$ by faotors of three. We have made provision for an harmonic cavity which can increase oz by a factor of about two. Also, we think that we can use an addition "experience factor" of two in Eq. (19) as previously noted. We cannot reasonably decrease the integral $\&$ by either an increas? in the Touschek $n$ factor or in $a_{p}$ unless our oalculation of beam energy spread is in error due to use of $V m=2.4 \mathrm{MV}$ at injeotion which we have oeen leads to an unrealistioally large energy buoket. Therefore, we conciude that the estimate of $\tau$ tou at $100 \mathrm{MeV}$ is marginal for injection.

One final remark on Touschek lifetime. We have intentionally avoided any discussion of the numerous transverse and longitudinal instabilities. This subject, endemic to all storage rings, could readily comprise a separate study and is germain to the present work only with regard to the effect of instabilities on beam size and hence touschek lifetime. Instabilities generally increase beam size, to the detriment of colliding beam experiments, but perhaps an advantage for this applioation by inoreasing $T$ tou.

\section{Preliminary Cost Estimate}

The results are given in Table VII. Here we will discuss each item separately, noting at the outset that this cost estimate does not include cost of a building and that the estimate for a possible Booster is done separately, as is the cost of a superconducting wiggler pius associated beam line. First, the ring magnet system is directiy scaled from the NSLS vUV ring $(E=0.7 \mathrm{GeV} L=51 \mathrm{~m})$ value of $\$ 445.9 \mathrm{~K}$ multiplied by four years of inflation at 10\%/year. The result, $\$ 652.8 \mathrm{~K}$, is well below the SLAC Damping ring $(E=1.2 \mathrm{GeV}, L=34 \mathrm{~m})$ estimate of $\$ 1.31 \mathrm{M}$, but their estimate includes power supplies and, more imperatively, an extensive transport system from the Linac to the Damping ring and back. Our estimate is also well below the LBL ALS ring ( $E=1.3 \mathrm{GeV}, L=182.4 \mathrm{~m}$ ) of $\$ 2.71 \mathrm{M}$; however, $L B L$ is using 84 quadrupoles rather than our 24 and 36 sextupole magnets compared to our 16. In general. we feel more comfortable in soaling from actual (NSLS) rather than estinated costs. 
The magnet power supplies are again direotly scaled from the NSLS \$151.2K value with a faotor of 1.464 inflation and an additional factor of 1.25 to account for our need for 4 rather than 3 quadrupole power supplies. The addition of the resulting $\$ 276$. $7 \mathrm{~K}$ brings us uloser to the SLAC result again, we are lower than the LBL ALS value of $\$ 645 \mathrm{~K}$ but they inolude one additional quadrupole power supply and 72 ring steering supplies (we will also need steering supplies, but a lesser number--32 as in the MSLS VUV--and include the itam under diagnostio equipment).

The ring vacuum system is one of the easier items; we will soale direotiy from the NSLS cost of $\$ 430 \mathrm{~K}$ since the rings are of comparable ciroumference; including the 1.464 inflation factor, the vacuum system estimate is \$630K.

For computer control the NSLS cost is $\$ 723.8 \mathrm{~K}$. However, this inoludes the cost of two larger control computers ( $O G$ EClipse) and at least 3 times the number of micro-computers (Intel $80 / 80$ ) for their 3 rings as will be needed here. It is unclear to us whether we will even require a larger computer (of the order of a VAX-11/780) for machine control but we strongly suspect that the analysis of the angiography data will require it. so we multiplied the NSLS number by 0.5 and then used the 1.464 inflation factor to obtain $\$ 529.8 \mathrm{~K}$.

The NSLS shielding costs also ircluded survey but we believe that the value of $\$ 1.511 \mathrm{M}$ is primarily shielding and will scale linearly with ciroumference. Their three rings have a total circumference of $249 \mathrm{~m}$ so we multiplied the NSLS number by $54 / 249$ and the 1.464 inflation faotor to obtain $\$ 479.9 \mathrm{~K}$.

For the rings diagnostio plus security system, the NSLS value is $\$ 464.4 \mathrm{~K}$. We have added an additional $\$ 30 \mathrm{~K}$ for the two main power supplies plus pole tip secondary windings of the orbit correction system. then multiplied by $\$ 1.945 M / \$ 7.79 M=0.25$ which is the fraction of the total capital cost expended on the vuV ring, and again used the 1.464 inflation factor to get $\$ 189.0 \mathrm{~K}$.

The total capital cost to this point is $\$ 2.75 \mathrm{M}$. The final capital item is the RF system. We note first that the low frequency ( 53 Mhz) NSLS $x$-ray ring RF system with power capability of approximateiy $700 \mathrm{KW}$ cost $\$ 611.6 \mathrm{~K}$ or, with the inflation factor, $\$ 895.4 K$. One of the factors affecting the choice of $R F$ irequency is certainly the lower cost of a $50 \mathrm{Mhz}$ compared to a 500 Mhz system. The trade-off may well be the factor of 10 higher Touschek lifetime at injection which the 500 Mhz system gives us if that factor is the one that determines whether or not a Booster syrichrotron is required. For the present We have no better estimate than the $\$ 1.765 M$ from the LBL proposal. Thus, the RF system is $\approx 40 \%$ of the capital cost of the machine. It was not possible here to give consideration to the rather low estimate of \$140K for the SLC Damping ring RF; that system is a high frequency one also, but low power- 16 KW for radiation plus cavity losses--and there were mitigating circumsiances in obtaining surplus UHF transmitting equipment.

The direct labor cost of construction for the NSLS VUV was $\$ 438.3 \mathrm{~K}$ and, with inflation, \$641.7K is used in Table VII. We have no reason to assume that this $i$ tem would be different elsewhere.

Finally, the design cost for the NSLS project, listed as Engineering Design and Inspeotion (EOI) is $23.5 \%$ of the tatal project capital cost or, in our case, $0.235 \times(\$ 4.515 \mathrm{M})=\$ 1.061 \mathrm{M}$. Then we add $10 \%$ contingeney of $\$ 621.8 \mathrm{~K}$ for an overall total of $\$ 6.84 M$ for the ring. 
In the present cost estimate of the facility we am assuming no Booster. Thus, the final item is the pre-injector and associate beam transport system to the ring. We choose a $100 \mathrm{MeV}$ microtron which cost $\$ 275 \mathrm{~K}$ at the University of Wisconsin.50 With a 5-vear inflation factor we obtain $\$ 443 K$. For the inicratron-to-ring transport system, we note that the NSLS cost for a 700 MeV transport system to the vuv ring was $\$ 321.5 \mathrm{~K}$. We assume that the present transport system will include as many dipoles (2) and quadrupoles (9), but that the cost scales with energy. Including the inflation factor, this gives $\$ 67.2 \mathrm{~K}$.

\section{Acknowledgements}

It is a pleasure to thank R. Hofstadter and E. B. Hughes for providing the facilities of the Hansen Laboratories of Physics at Stanford University, for helpful discussions and encouragement in the present work, and for suggesting an investigation of the problem of designing an $x$-rav source appropriate for clinical use. It is also a pleasure to acknowledge my gratitude to $H$. Winick and $A$. Bienenstock for providing the facilities of the stanford synchrotron Radiation Laboratory and to M.J. Lee and M. Woodley for making available the SLAC linear lattice design code COMFORTS which was used for much of the study, to S. Goldberg for getting the program running on the SSRL VAX, and to R.Z. Liu for use of his lattice search program LAMP. We thank G. Brown of SSRL and S. Krinsky of BNL for important advice on wiggler spectra, and W. Foyt of BNL for providing the budget data on which the present cost estimate is largely based. We would indeed be remiss if we failed to acknowledge the considerable programming effort of my wife, $S$. Blumberg, for all programs used in this report except the above mentioned lattice design codes. It is indeed a pleasure to thank $G$. Hinshaw of the Hansen Laboratories of Physics at Stanford University, for her excellent work in typing and editing this manuscript. Finally, we wish to thank M. Blume, J. Mctague and A. van steenbergen for making possible this leave of absence from BNL to work with the Stanford Angiography group. 


\section{REFERENCES}

1. E. Rubenstein,

E.B. Hughes,

L.E. Finman,

R. Hofstadter,

R.L. Kirk,

T.J. Krolicki.

J.P. Stone

S. Wilson,

H.O. Zeman.

W.R. Brody,

A. Macovski, and A.C. Thompson, sunchrotron Radiation and its Application to Digital Subtraction Angiography, SPIE, 314 (1981).

2. Ibid

3. W.R. Dix, C.C. Glüer, W. Graeff, K.H. Höhne and W. Kupper, Deutsches Elektronen-Synchrotron report OESY-SR-82/24 (1982).

4. Proposal for a National Synchrotron Light Source, ed. J.P. Blewett, $B N L-50595$ (1977).

5. Design study for a Dedicated Source of synchrotron Radiation, D.J. Thompson, et. al. Daresbury (U.K.) report OL/SRF/R2 (1975).

6. S. Kamada, Y. Kamiya and M. Kihara, National Laboratory for High Energy Physics KEK (Japan) report KEK-77-16 (1977).

7. Stanford Linear Accelerator Center report SLAC-229 (1980).

8. H.C.H. Hsieh, private communication, 1982.

9. Spectra and Optics of Sunchrotron Radiation. G.K. Green, BNL-50522, Eq. (22), p.9 (1976). See also J. Schwinger, Phys. Rev. 75,1912 (1949).

10. G. Brown, K. Halbach, J. Harris and H. Winick, Proc. Int. Conf. on $x-r a y$ and VuV Synch. Rad. Instr., Hamburg (1982) to be published in Nucl. Instr. $\varepsilon$ Meth.: S. Krinsky, W. Thomlinson and $A$. van Steenbergen, BNL-31989 (1982).

11. V.P. Suller, N. Marks, M.W. Poole and R.P. Walker, Proc, of the U.S. Particle Accel Conf., Santa Fe, NM (1983) and private communication (1983).

12. Matsushita, Kitamura and lshikawa, private communication (March, 1983).

13. J. Galayda, et.al., Proc. of the U.S. Particle Accel. Conf.. Santa Fe, NM (1983). To be published in IEEE Trans. Nucl. Sci.

14. J.D. Jackson, Classical Electrodynamics (2nd edition), J. Wiley E Sons, Inc., (1975), chapt. 14, Eq. (14.88). Note that Jackson defines the critical frequency as $\omega_{c}=3 \gamma^{3}(c / \rho)$ which is a factor of 2 larger than as defined by schwinger ( $r e f .8$ ) and used by all other references cited in this report. We use the schwinger definition in this report.

15. Suggested independently by myself (1982) and $V$. Kulipanov of the Novosibirsk group and communicated to E.8. Hughes (1982).

16. S. Krinsky, W. Thomlinson, and A. van steenbergen, BNL-31989 (1982). p.27, Table 4.1.

17. See, for example, L. Blumberg, et. al. Brookhaven National Laboratory report AGS-71/02 and IEEE Trans. Nucl. Sci. NS-18, 1009 (1971).

18. E.D. Courant and H.S. Snyder, Ann. Phys. 3, 1 (1958).

19. This result was communicated to me by C. Pellegrini (1981). A similar result is obtained by J. Leduff, Laboratoire de l'Accelerateur Lineaire report LAL 82,42, orsay, France (1982); however, LeDuff's result is missing a factor of $a / \beta$ s.

20. A good discussion of this process is contained in $H$. Bethe and J. Ashkin, Experimental Nuclear Dhysics (ed E. Segre), Vol. I, Part II. p. 2G0. Eq. (57). J. Wiley and Sons, Inc. (1953). An application to the BNL NSLS rings is given in L. Blumberg and M. Perlman, NSLS Tech. Memo (!98n!)

21. J. Harris, private communication (1983).

22. Y. Yan and R.Z. Liu, private communication, 1983 and Y. Yan, Proc. of the 
U.S. Particle Accel Conf., Santa Fe, NM (1983).

23. E. Rowe, private communication (1983).

24. M. Sands, The Physics of Electron Storage Rings, stanford Linear Accelerator Report, SLAC-121 (1970).

25. A good discussion of the rouschek Effect can be found in H. Bruch, Circular Particle Accelerators, (translation) Los Alamos National Laboratory report LA-TR-72-10 (1972). Chapt. XXX.

26. H. Wiedemann, stanford Linear Accelerator Center internal report PEPNOTE-27 (1973). The subject has also been treated by V. Volkel, Deutsches Elektronen-synchrotron internal report DESY-67/5 (1967) and 8. Gittelman and D.M. Ritson, Stanford University, Physics Dept. internal report HEPL-299 (1963).

27. A.S. King and M.J. Lee, SLAC internal report SPEAR-201 (1977) and reference 24 .

28. R. Heese, private communication (1980).

29. J. Allinger and J. Jackson. private communication ( 1981 ).

30. M.J. Lee and M. Woodley, private communication (1982).

31. A.S. King, M.J. Lee and W.W. Lee, Stanford Linear Accel. Center report SLAC- 183 (1975).

32. R.Z. Liu, private communication (1983).

33. See, for example, L. Blumberg, et.al., IEEE Proc. Nucl. Sci. NS-26, 3842 (1979) and BNL-25777 (1979).

34. See, for example, L. Blumberg, SSRL informal report SSRL-TM-83/02 (9983) and references therein such as H. Wiedemann, SLAC informal report PEP$T N-220(1981)$.

35. See, for example, L. 8lumberg, BNL informal note NSLS-TH-16 (1979).

36. M. Plotkin. private communication (1979).

37. S. Krinsky, et al., IEEE Proc. Nucl. Sci. NS-26, 3806, (1979).

38. L.N. Blumberg and R.Z. Liu. SSRL informal report SSRL-TN-83/01 (1983).

39. J. Harris, private communication (1983).

40. Conceptual Design Report for the National Center for Advanced Materials, Vol. I. LBL informal report PUB-5084 (1983).

41. H. Wiedemann, private communication (1978).

42. J. Kouptsidis and A.G. Mathewson, Deutsches Elextronen-synchrotron internal report DESY $76 / 49$ (1976).

43. A.G. Mathewson, G. Horikoshi and H. Mizuno. Mational Laboratory for High Energy Physics KEK (Japan) report KEK-78-9 (1978).

44. J.C. Schuchman, BNL NSLS report BNL-32167 (1982).

45. J. LeDuff, Laboratoire de l'Accelerateur Lineaire (Orsay) report LAL $82 / 42$ (1982).

46. J. Harris and R. Stege, private communication (1983).

47. E. Rowe, private communication (1983).

48. NSLS Design Handbook, Chapt. 3, P. 37, Table 3.3-I, unpublished (1978).

49. W. Foyt, private communication (1982).

50. E. Rowe, private communication (1983). 
TABLE II. Parameters of $L=53.96 \mathrm{~m}, h=90,15^{\circ}$ Dipole Lattice
A. $5 / 29-16$ B. $5 / 29-20$
C. $5 / 29-23$
D. $6 / 1-3$
E. $6 / 1-4$
F. 6/1-5 G. 6/1-6

$g_{Q F 1}\left(m^{-2}\right)$

$1.87364 \quad 1.84578$

1.78643

4.15900

4.12846

4.08471

4.95437

$\mathrm{J}_{Q D 1}\left(\mathrm{~m}^{-2}\right)$

$-2.77610$

$-2.56596$

$-2.18089$

$-3.29873$

$-2.87862$

$\begin{array}{ll}-2.72317 & -2.37643\end{array}$

${ }^{9} \mathrm{QF} 2\left(\mathrm{~m}^{-2}\right)$

2.51201

2.52811

2.57074

3.58612

3.41876

3.12434

2.85113

$\mathrm{g}_{\mathrm{QD} 2}\left(\mathrm{~m}^{-2}\right)$

1.25049

1.24557

$1.22020 \quad-.35989$

0.11203

$0.71130 \quad-1.11724$

$g_{Q F 3}\left(m^{-2}\right)$

\begin{tabular}{|c|c|c|c|c|c|c|c|}
\hline$v_{x}$ & $(3.25)$ & $(3.25)$ & $(3.25)$ & $(5.25)$ & $(5.25)$ & $(5.25)$ & $(5.25)$ \\
\hline$v_{Y}$ & $(3.25)$ & (3.25) & $(3.25)$ & $(3.25)$ & $(3.25)$ & $(3.25)$ & $(3.25)$ \\
\hline$B_{x}^{*}(m)$ & 11.59 & 10.95 & 9.77 & .29 & .29 & .28 & $(.5)$ \\
\hline$B_{y}^{*}(m)$ & .79 & .71 & .02 & 1.24 & 1.35 & .06 & 1.71 \\
\hline$n_{x}^{*}(m)$ & $(0)$ & $(0)$ & $(0)$ & $(0)$ & $(0)$ & $(0)$ & -1.0 \\
\hline$B_{x}(m)$ & 11.87 & 10.96 & 10.10 & 11.72 & 11.49 & 12.24 & 17.12 \\
\hline$B_{y}(m)$ & 29.14 & 23.30 & 19.98 & 47.74 & 42.56 & 36.02 & 53.80 \\
\hline$\hat{n}_{x}(m)$ & 1.35 & 1.32 & 1.26 & 1.28 & 1.23 & 1.21 & 4.90 \\
\hline$\xi_{x}^{0}$ & -3.77 & -3.55 & -3.16 & & & & -5.43 \\
\hline$\xi_{y}^{o}$ & -11.93 & -9.81 & -19.50 & & & & -18.54 \\
\hline$U_{0}^{(1.4)}(\mathrm{keV})$ & 133.4 & 641.6 & 2166.0 & & & & \\
\hline$u_{0}^{(.1)}(\mathrm{keV})$ & & & .056 & & & & .017 \\
\hline${ }^{\alpha} p$ & .072 & .070 & .067 & .069 & .067 & .068 & .27 \\
\hline$\psi_{k} / 2 \pi$ & .270 & .266 & .256 & .27 & .27 & .27 & .40 \\
\hline$B_{x}^{k}(m)$ & 1.53 & 1.54 & 1.58 & 1.84 & 1.87 & 2.05 & .31 \\
\hline$\tau^{(1.4)}(\mathrm{ms})$ & 3.75 & .78 & .23 & & & & \\
\hline$\tau_{x}^{(.1)}(\mathrm{ms})$ & & & 634.5 & & & & 215 \\
\hline$\dot{\sigma}_{x}^{\star(1.4)}(\mathrm{mm})$ & 1.86 & 1.02 & 1.71 & & & & \\
\hline$\sigma_{-x}^{*}(.1)(\mathrm{mm})$ & & & .12 & & & & .10 \\
\hline$\sigma_{z}(1.4)(\mathrm{mm})$ & 11.20 & 21.4 & 47.0 & & & & \\
\hline$\sigma_{z}^{(.1)}(\mathrm{mm})$ & & & .59 & & & & \\
\hline$\frac{{ }^{6}}{E} \times 10^{4}(1: 4)$ & 7.6 & 14.4 & 21.7 & & & & \\
\hline$\frac{\sigma_{E}}{E} \times 10^{4}(0.1)$ & & & 1.5 & & & & \\
\hline
\end{tabular}




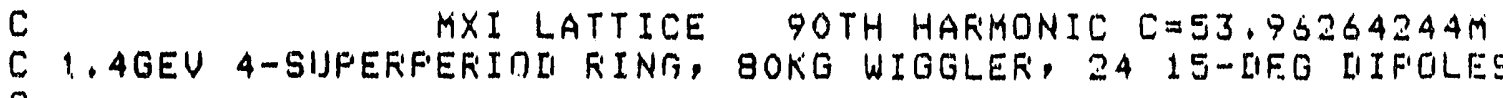

C

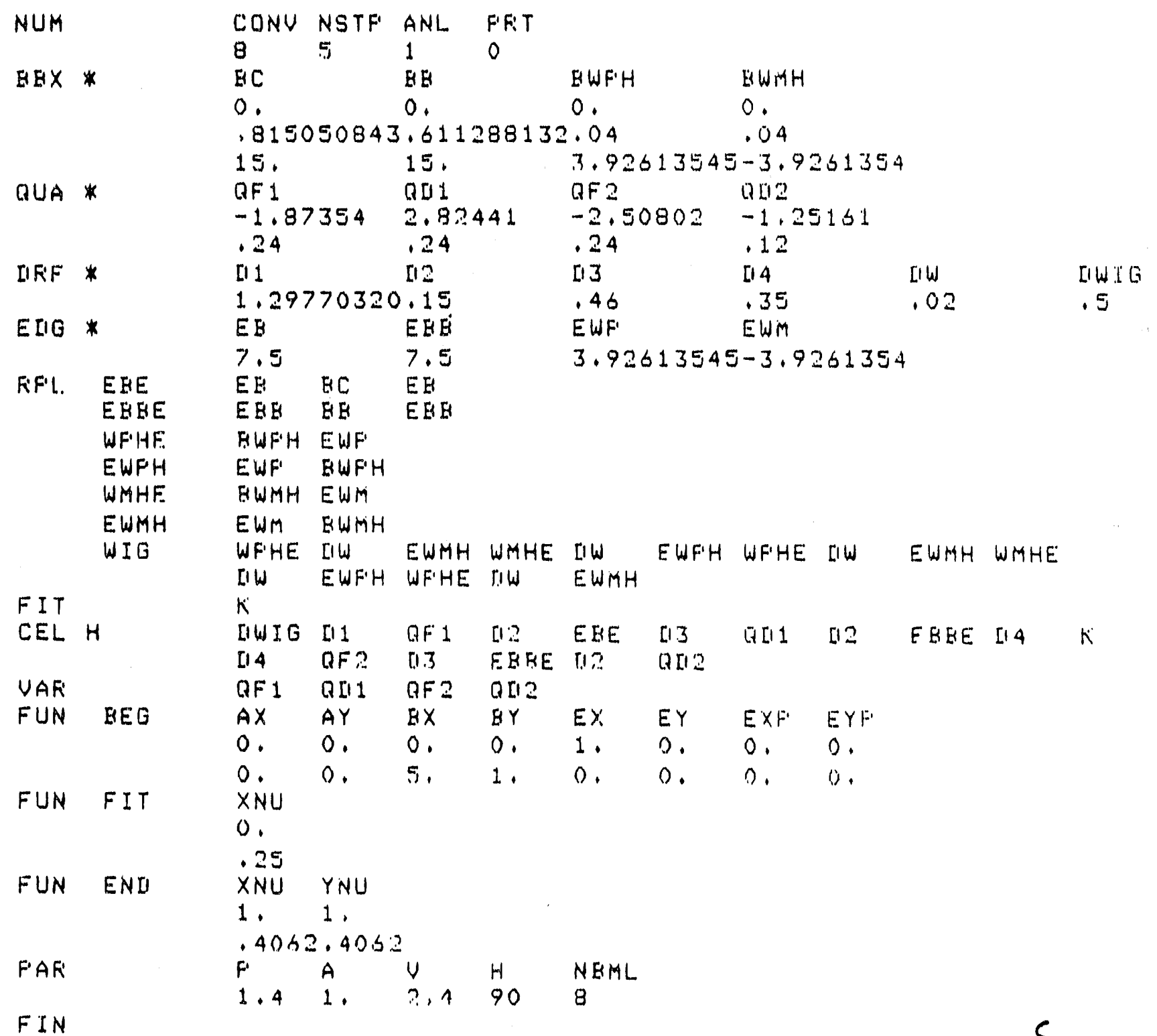

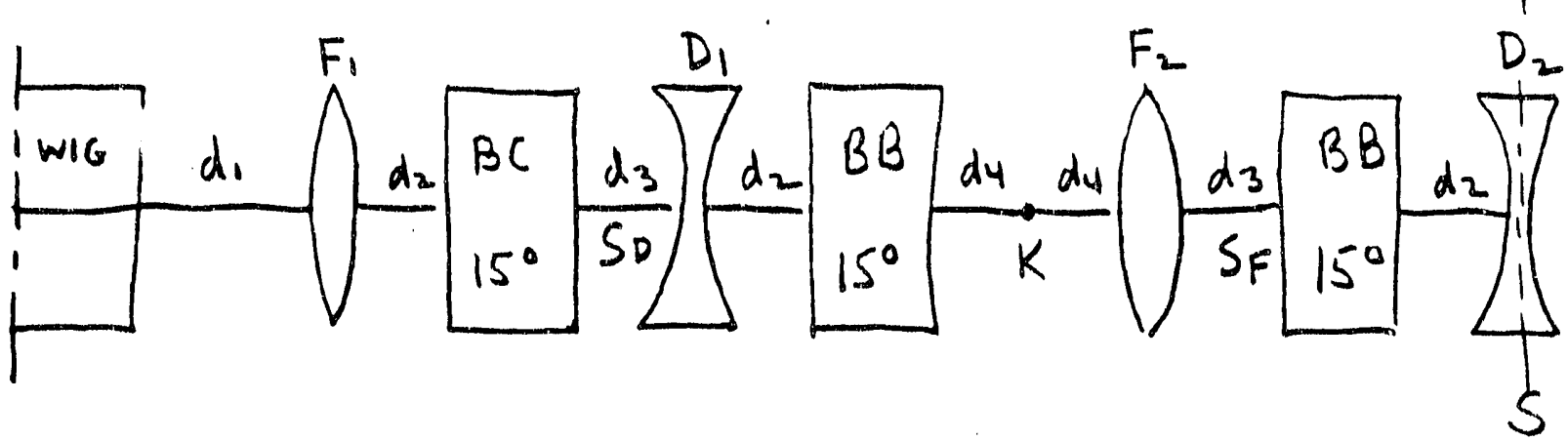

TABLE III. INPUT DATA FOR PROGRAM COMFORTS FOR SOLUTION IIA 5/29-16 


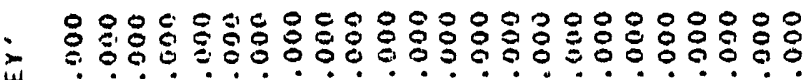

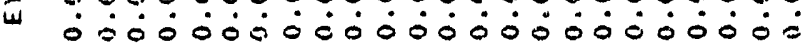

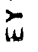

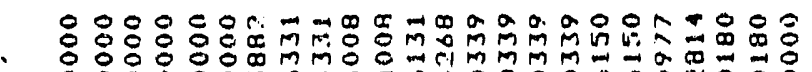

㐫

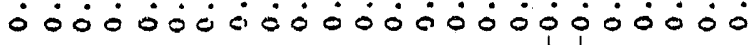

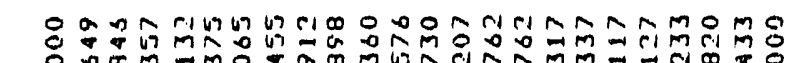

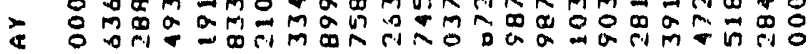

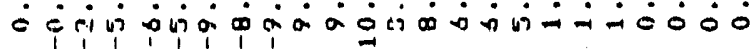

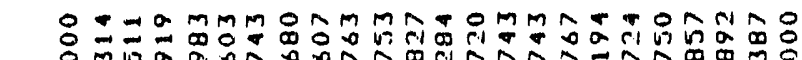

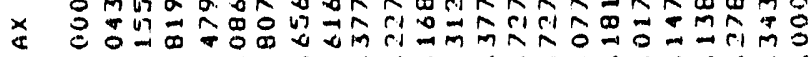

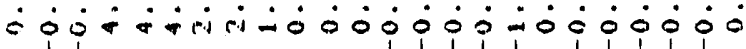

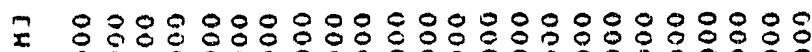

w

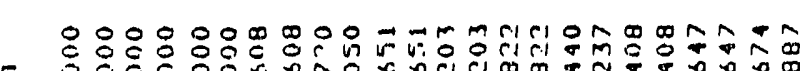

₹

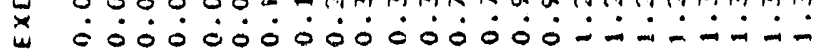

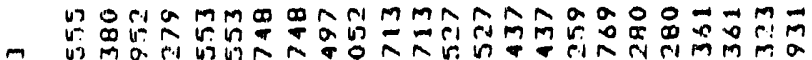

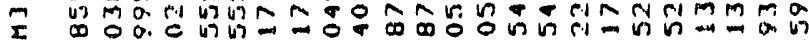

I

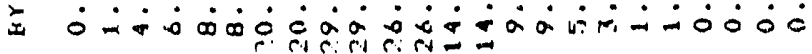

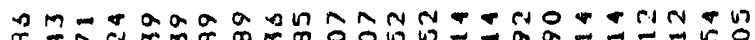

$\rightarrow$ a m $\rightarrow$ ricla

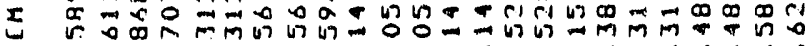

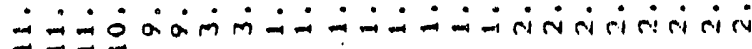

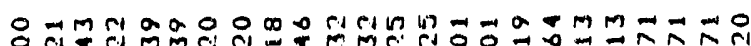

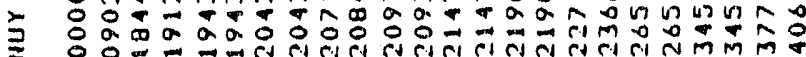

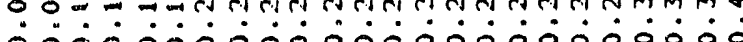

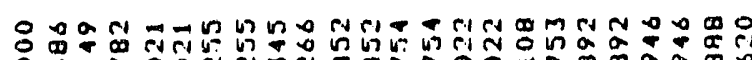

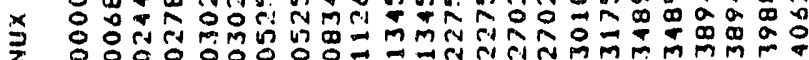

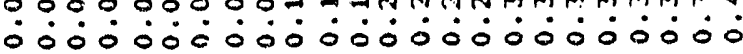

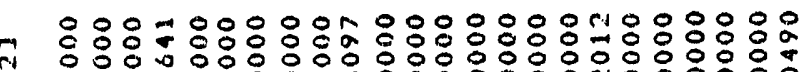

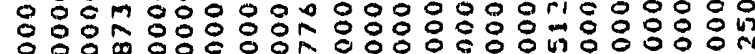

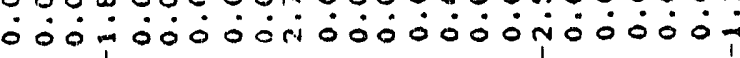

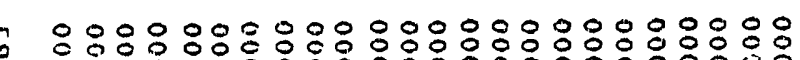
연.

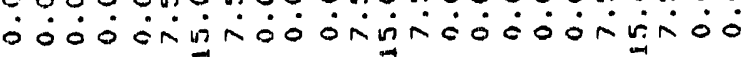

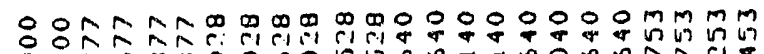

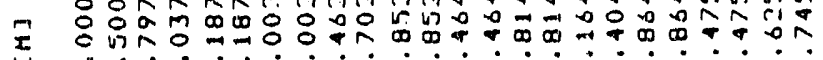
$\stackrel{x}{4}$

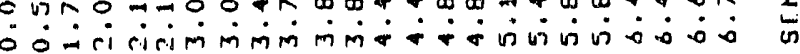

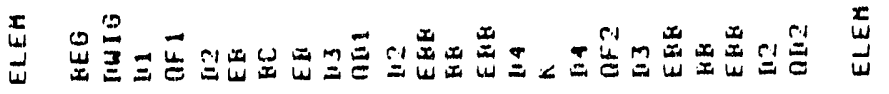




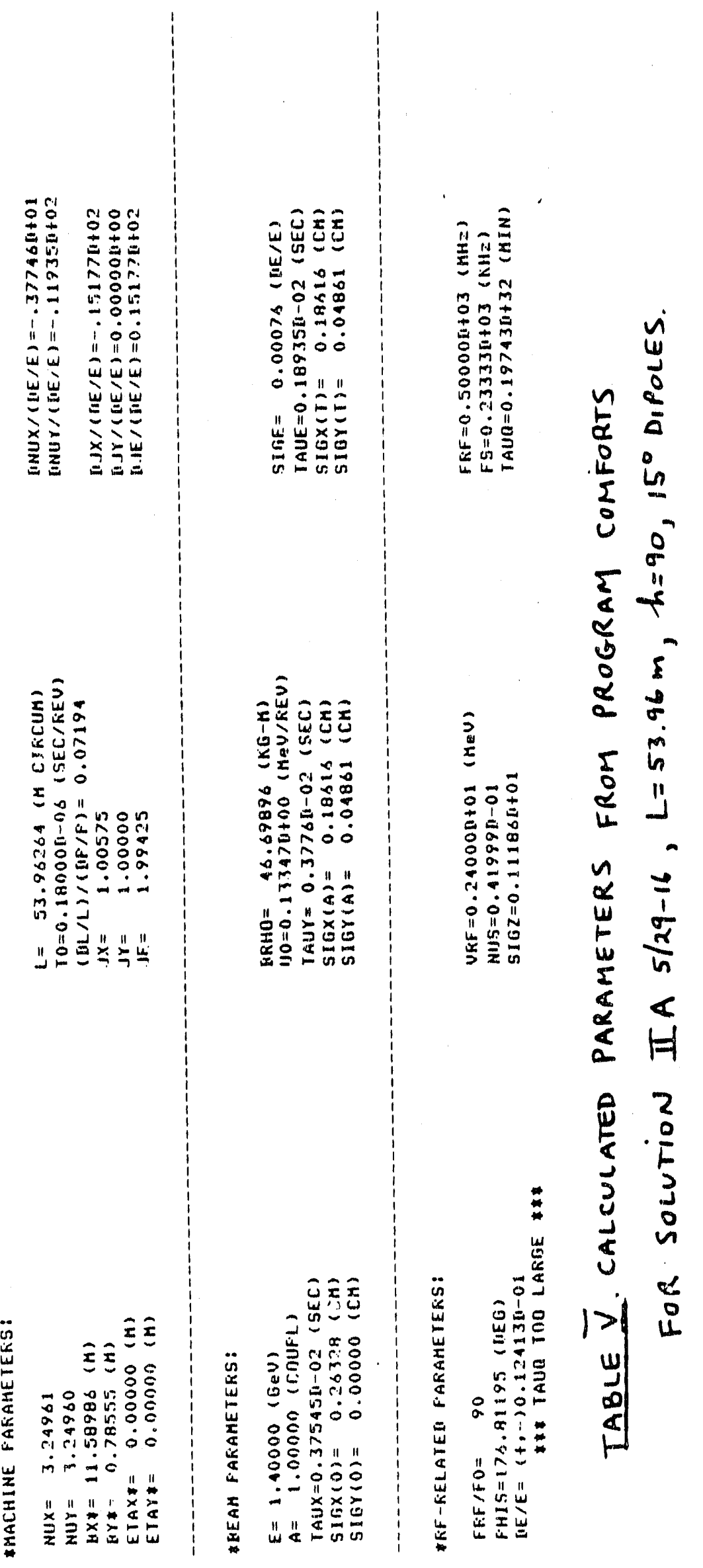


TABLE VI. Parameters of the $L=58.76 \mathrm{~m}, h=98,11.25^{\circ}$ Dipole Lattices

A. $5 / 24-1 \quad$ B. $6 / 1-9 \quad$ C. $5 / 26-1 \quad$ D. $5 / 26-2 \quad$ E. $5 / 24-4 \quad$ F. $5 / 26-4$ G. $5 / 26 / 11$

\begin{tabular}{|c|c|c|c|c|c|c|}
\hline$g_{0 F 1}\left(m^{-2}\right)$ & 0.65902 & 0.94059 & 3.12421 & 3.59028 & 4.36722 & 4.91320 \\
\hline
\end{tabular}

\begin{tabular}{llllllll}
\hline $\mathrm{g}_{\mathrm{QD} 1}\left(\mathrm{~m}^{-2}\right)$ & -2.74342 & -2.62454 & -3.39039 & -3.55676 & -4.67515 & -3.71578 & -3.83018
\end{tabular}

\begin{tabular}{llllllll}
\hline$g_{Q F 2}\left(m^{-2}\right)$ & 3.73336 & 3.68575 & 4.77114 & 4.21461 & 1.16638 & 5.20274 & 4.55218
\end{tabular}

\begin{tabular}{llllllll}
\hline $\mathrm{g}_{\mathrm{QD2}}\left(\mathrm{m}^{-{ }^{2}}\right)$ & -1.24726 & -1.49245 & -1.75813 & -1.95343 & -5.16879 & -1.24817 & -0.18811 \\
\hline $\mathrm{g}_{\mathrm{QF} 3}\left(\mathrm{~m}^{-2}\right)$ & 2.87698 & 3.08889 & 2.17921 & 4.05342 & 4.71748 & 0.75634 & 1.17242
\end{tabular}

\begin{tabular}{|c|c|c|c|c|c|c|c|}
\hline$v_{x}$ & $(3.25)$ & $(3.25)$ & $(5.25)$ & $(5.25)$ & $(5.25)$ & $(7.25)$ & $(7.25)$ \\
\hline$v_{y}$ & $(3.25)$ & $(3.25)$ & $(3.24)$ & $(3.25)$ & $(5.25)$ & $(3.25)$ & $(3.29)$ \\
\hline$\beta_{x}^{*}(m)$ & 3.25 & 3.73 & 4.93 & 1.51 & .12 & .20 & .50 \\
\hline$\hat{B_{y}^{\star}}(m)$ & $(.5)$ & $(.5)$ & $(1.0)$ & $(1.0)$ & 1.76 & 1.12 & $(1.00)$ \\
\hline$n_{x}^{*}(m)$ & $(0)$ & $(0)$ & $(0)$ & $(0)$ & -.12 & .03 & $(0)$ \\
\hline$B_{x}(m)$ & 20.04 & 13.86 & 5.67 & 5.37 & 43.35 & 18.33 & 31.37 \\
\hline$\hat{B}_{y}(m)$ & 27.69 & 20.23 & 32.32 & 36.40 & 31.65 & 46.41 & 72.32 \\
\hline$n_{x}(m)$ & 1.10 & 1.17 & .86 & .90 & 5.69 & .78 & .86 \\
\hline$\xi_{x}^{\circ}$ & -7.35 & -5.14 & -6.96 & -4.76 & & & -24.14 \\
\hline$\xi_{y}^{\circ}$ & -11.53 & -9.02 & -14.89 & -16.15 & & & -34.53 \\
\hline$u_{0}^{(1.4)}(\mathrm{keV})$ & 136.5 & 644.7 & 136.5 & 136.5 & & & 136.5 \\
\hline$u_{0}^{(.1)}(\mathrm{keV})$ & & 2336 & & & & & \\
\hline$a_{p}$ & .053 & .053 & .052 & .052 & .144 & .036 & .043 \\
\hline$\Psi_{k} / 2 \pi$ & .16 & .16 & .43 & .37 & .47 & .68 & .46 \\
\hline$B_{x}^{k}(m)$ & 15.26 & 10.77 & 4.79 & 1.56 & .58 & 6.84 & .17 \\
\hline$\tau_{x}^{(1.4)}(\mathrm{ms})$ & 4.01 & .85 & 4.01 & 4.01 & & & 4.01 \\
\hline$\tau_{x}^{(.1)}(\mathrm{ms})$ & & 2331.5 & & & & & \\
\hline$\sigma_{x}^{*(1.4)(\mathrm{mm})}$ & 1.75 & .78 & .75 & .52 & & & .42 \\
\hline$\sigma_{x}^{\star}(.1)(\mathrm{mm})$ & & .056 & & & & & \\
\hline$\sigma_{2}^{(1.4)}(\mathrm{mm})$ & 10.07 & 19.40 & 9.20 & 10.00 & & & 9.09 \\
\hline$\sigma_{2}^{(.1)}(\mathrm{mm})$ & & .021 & & & & & \\
\hline$\frac{E}{E} \times 10^{4}(1.4)$ & 7.7 & 14.4 & 7.7 & 7.7 & & & 7.7 \\
\hline
\end{tabular}

$\frac{\sigma_{E}}{E} \times 10^{4}(0.1)$

PIER

OFF

1.0

$3.926^{\circ} \quad$ OFF

OFF

OFF

OFF

OFF 
TABLE VII. Preliminary Cost Estimate of Medical X-Ray Source (in thousands of dollars)

I. Storage Ring

A. Magnet system (24 dipole, 28 quadrupole and 16 sextupole magnets)

$\$ 652.8$

B. Magnet power supplies (1 dipole, 4 quadrupole, 2 sextupole)

C. Vacuum System 630.0

D. Computer control 529.8

E. Shielding/Survey/Misc. (including cooling water and concrete pad of machine)

479.9

F. Beam diagnostics (including securtty system, transverse and longitudinal feedback components)

G. Ring RF system

$1,765.0$

total capital $\$ 4,515.2$

H. Associated labor and misc.

641.7

I. Eng. Design \& Inspect. (EDI)

$1,061.0$

ring sub total

$\$ 6,217.9$

J. Contingency at $10 \%$

621.8

total for Storage king

$\$ 6,839.7$

II. Pre-Injector (100 MeV Microtron)

$\$ 443.0$

Microtron-to-ring transport system

67.2

overall facility cost

$\$ 7,349.9$ 


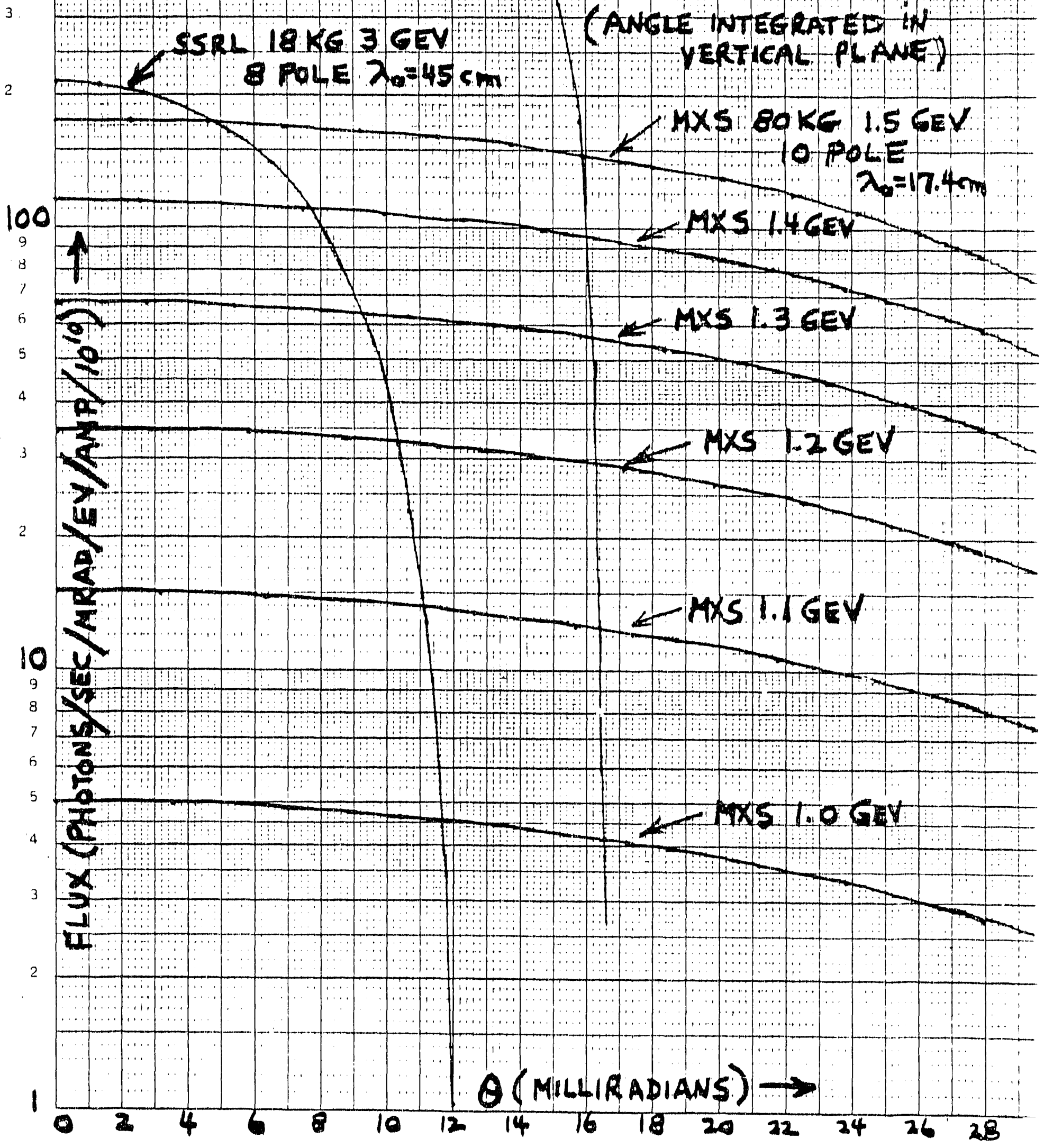




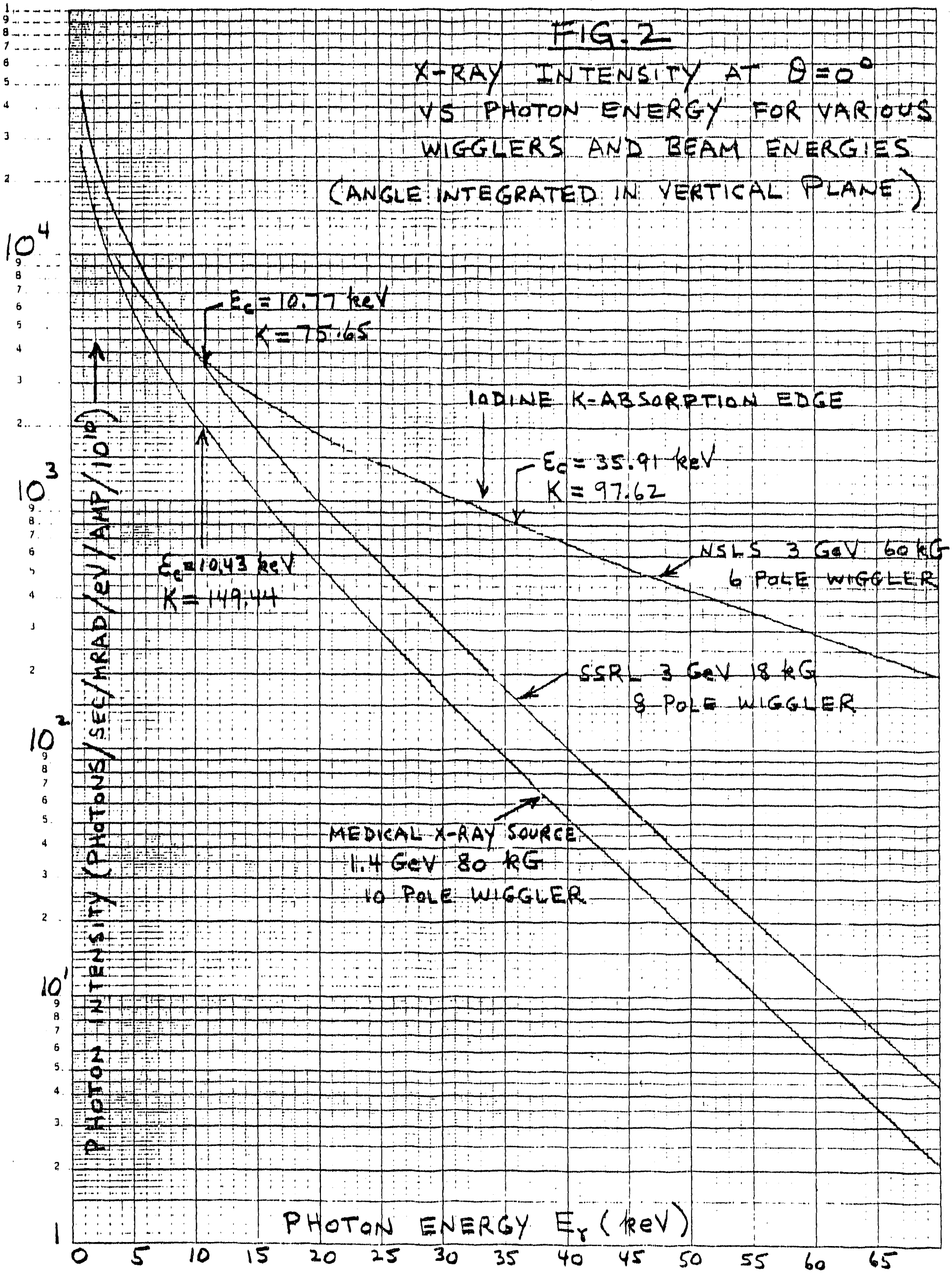




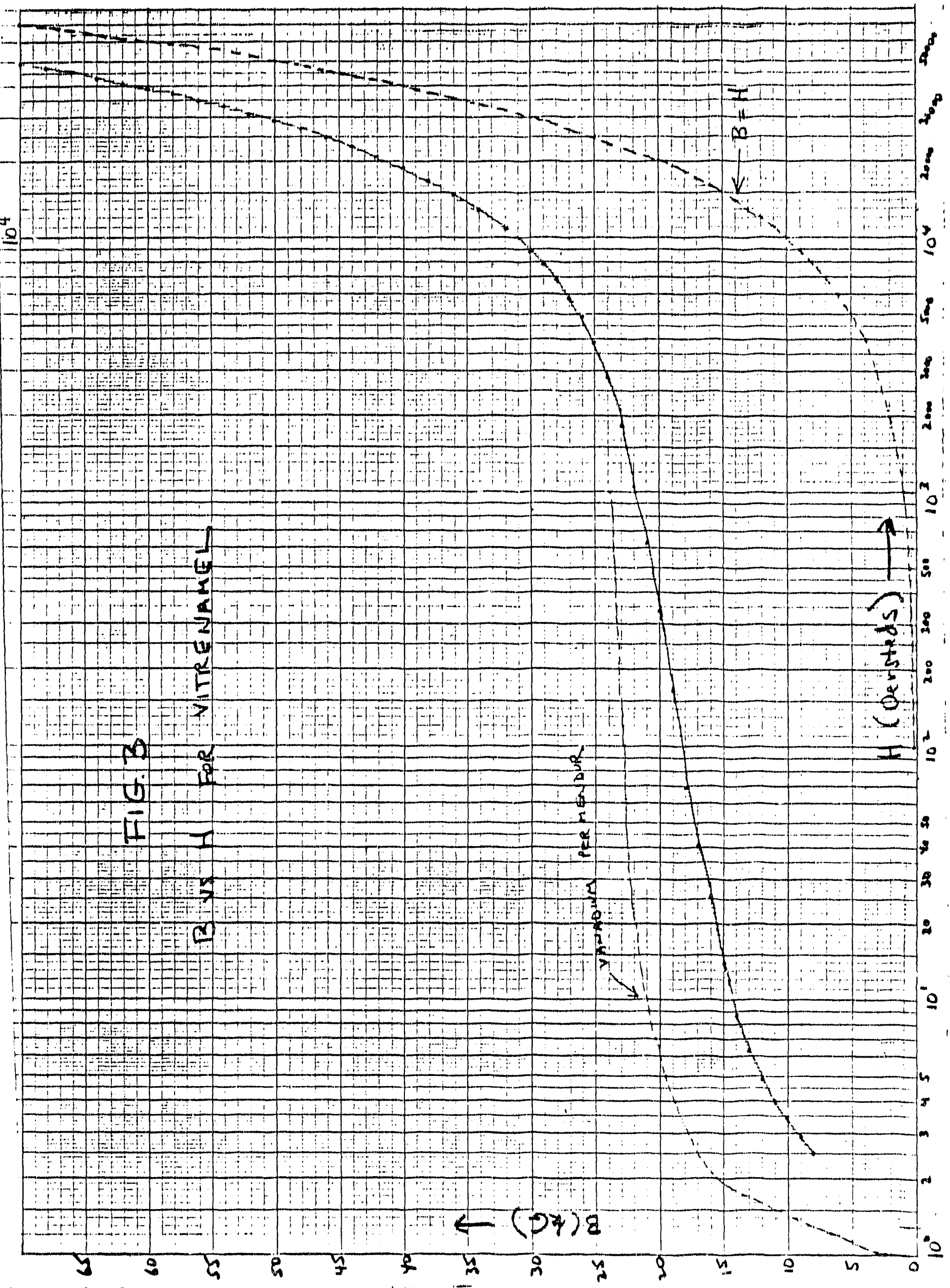




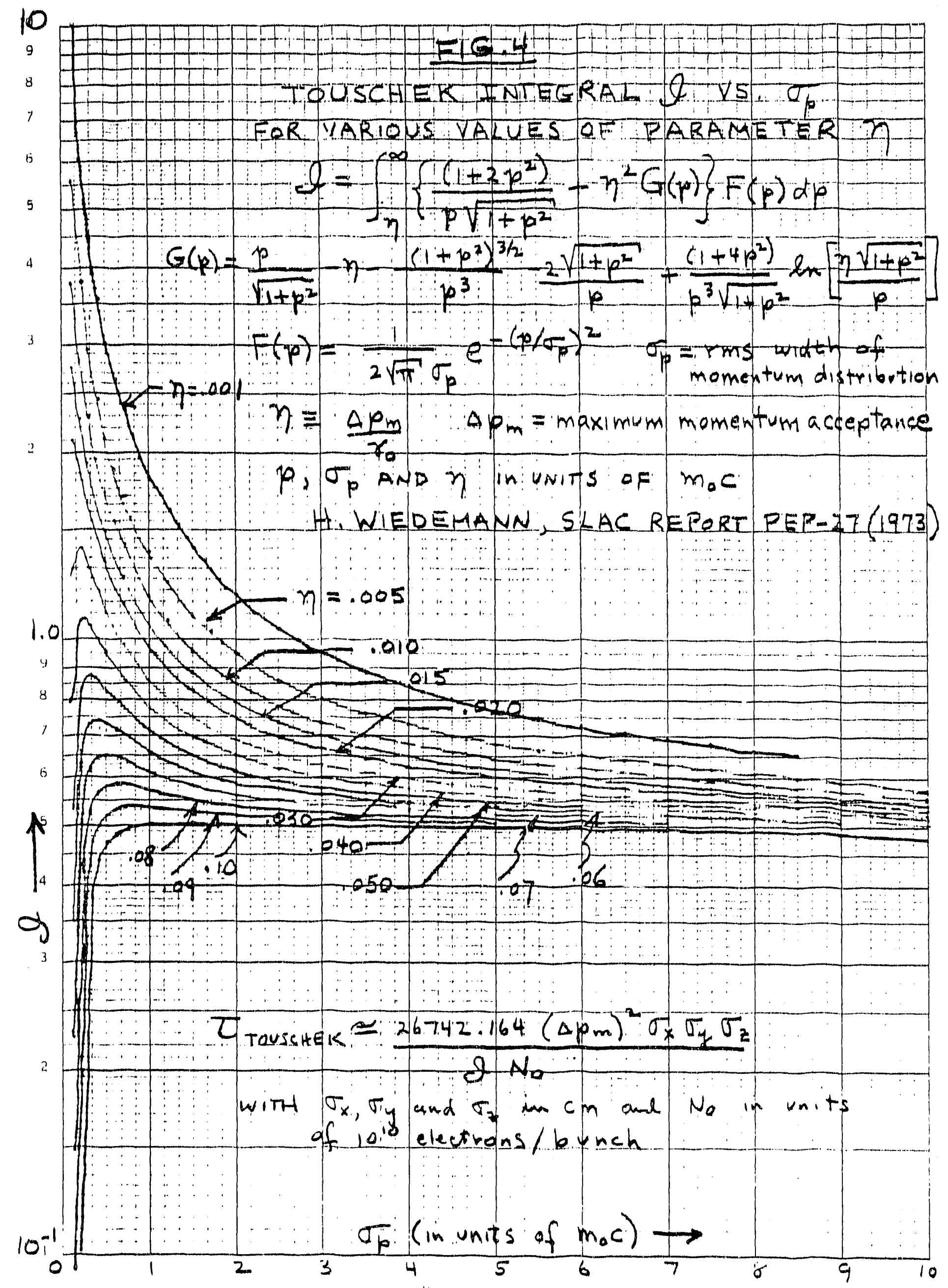




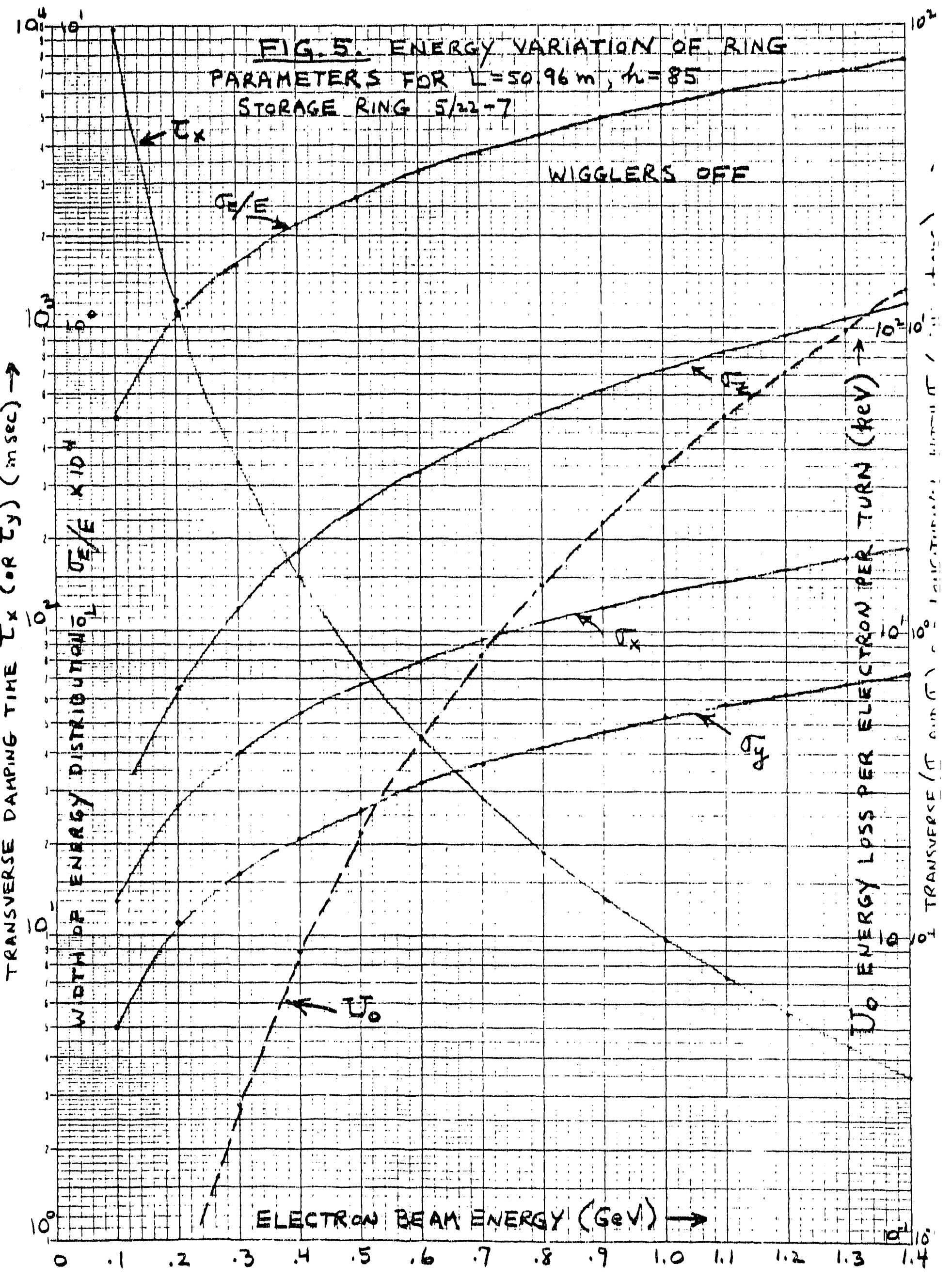




\section{$10 \frac{4}{10}$}

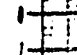

( IRTNG PARANETERS FOR L $=50.96 \mathrm{~m}$ h $=85$

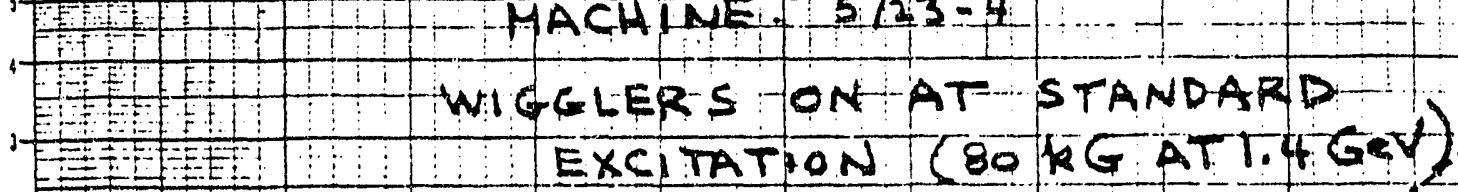

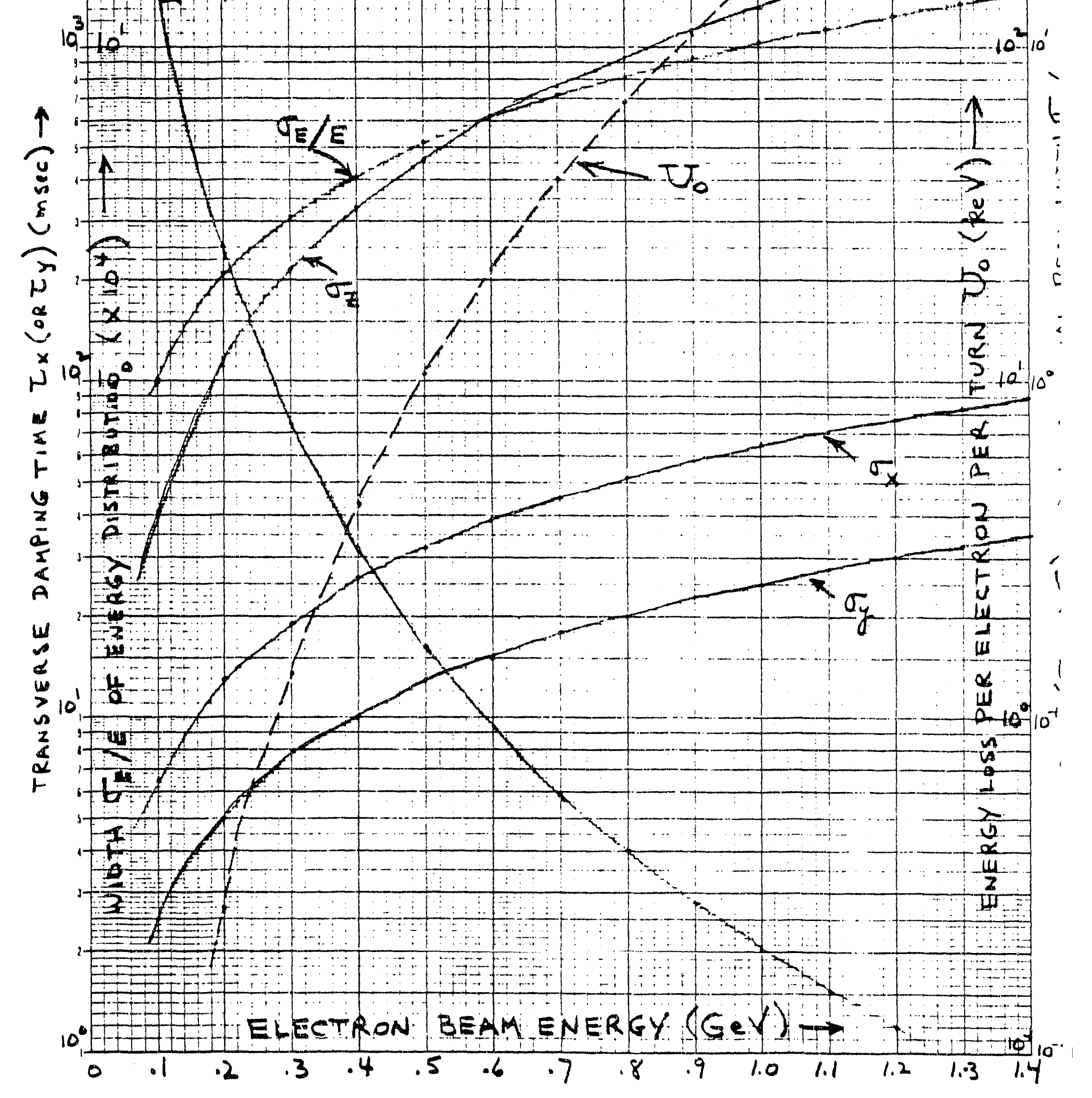




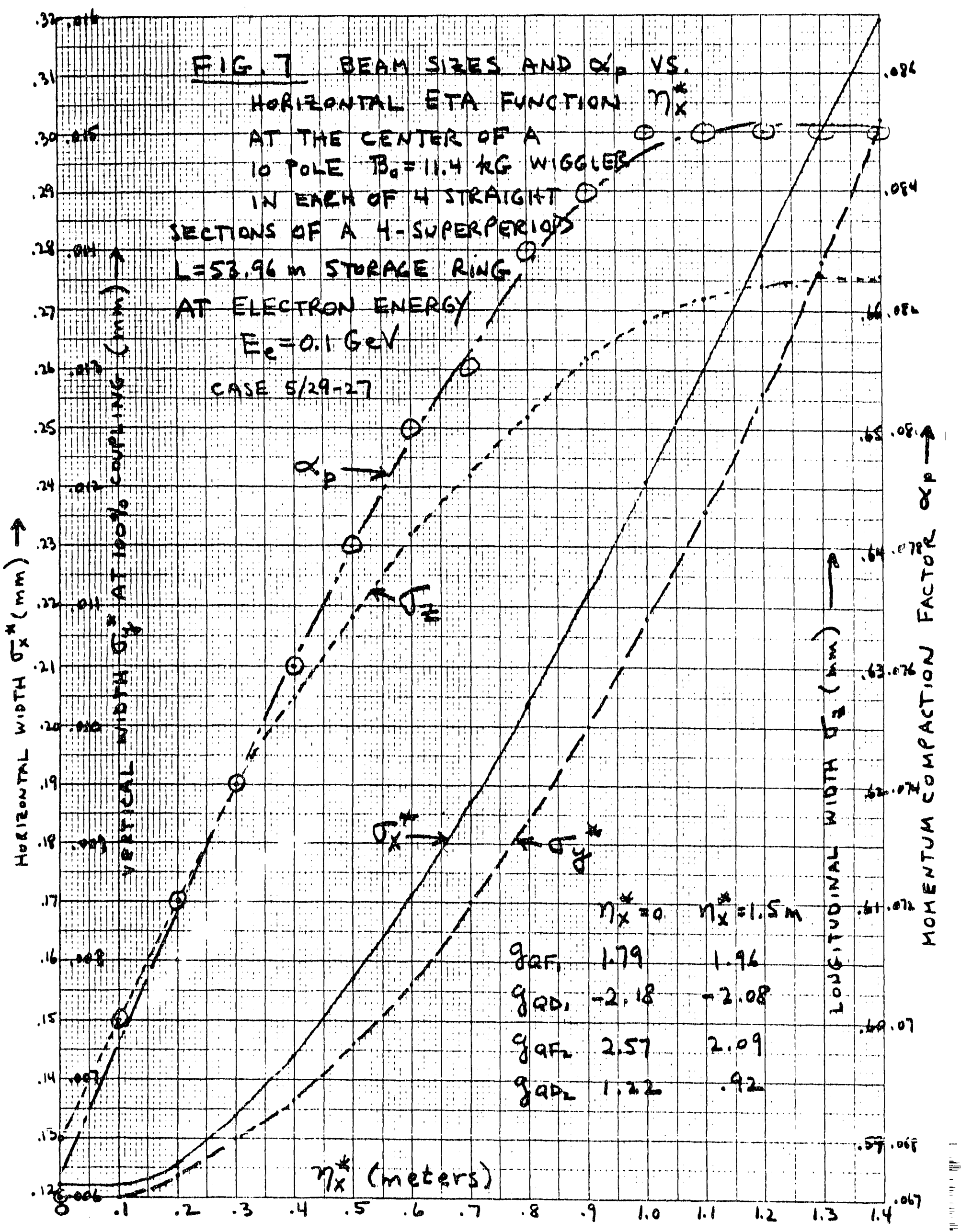



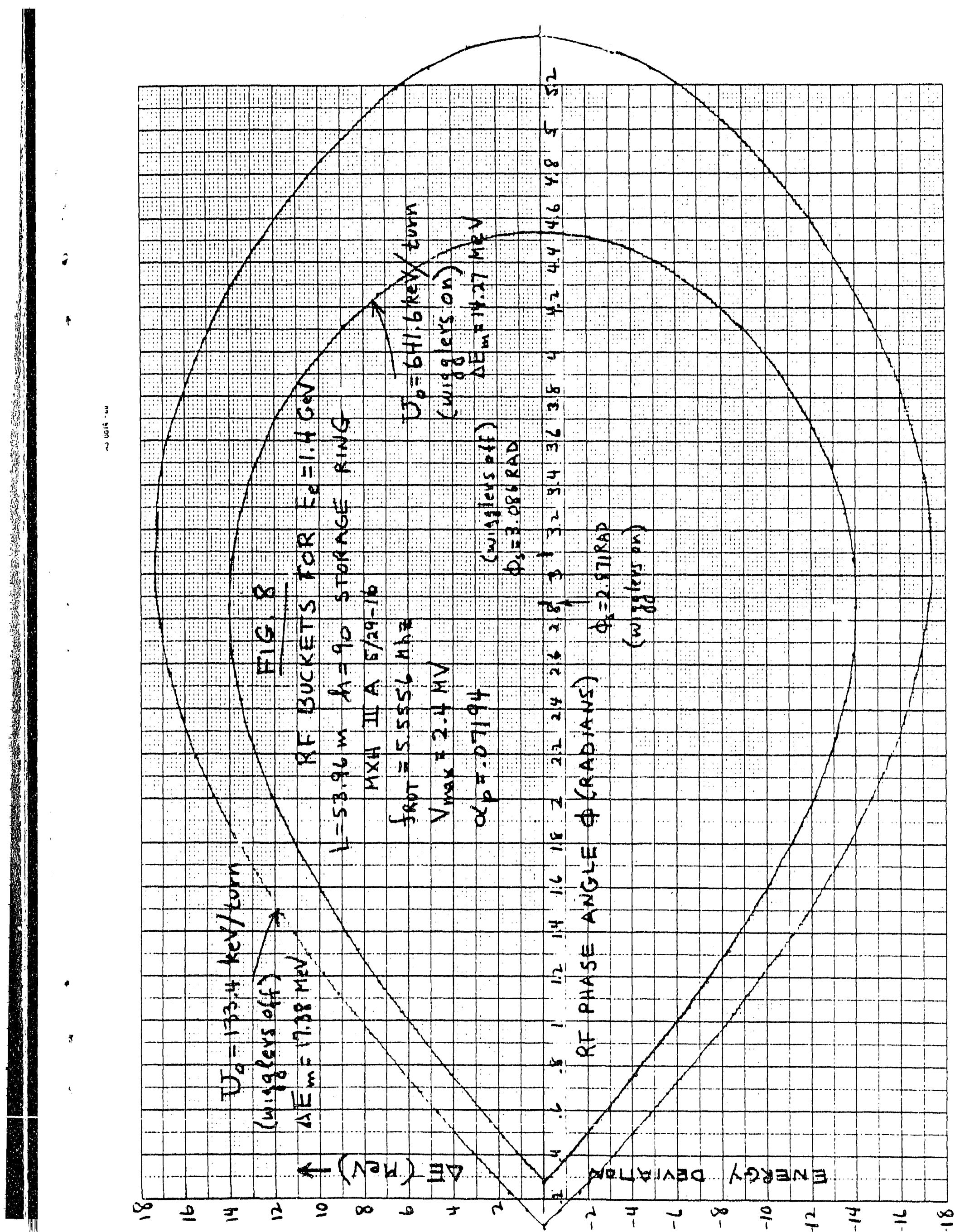

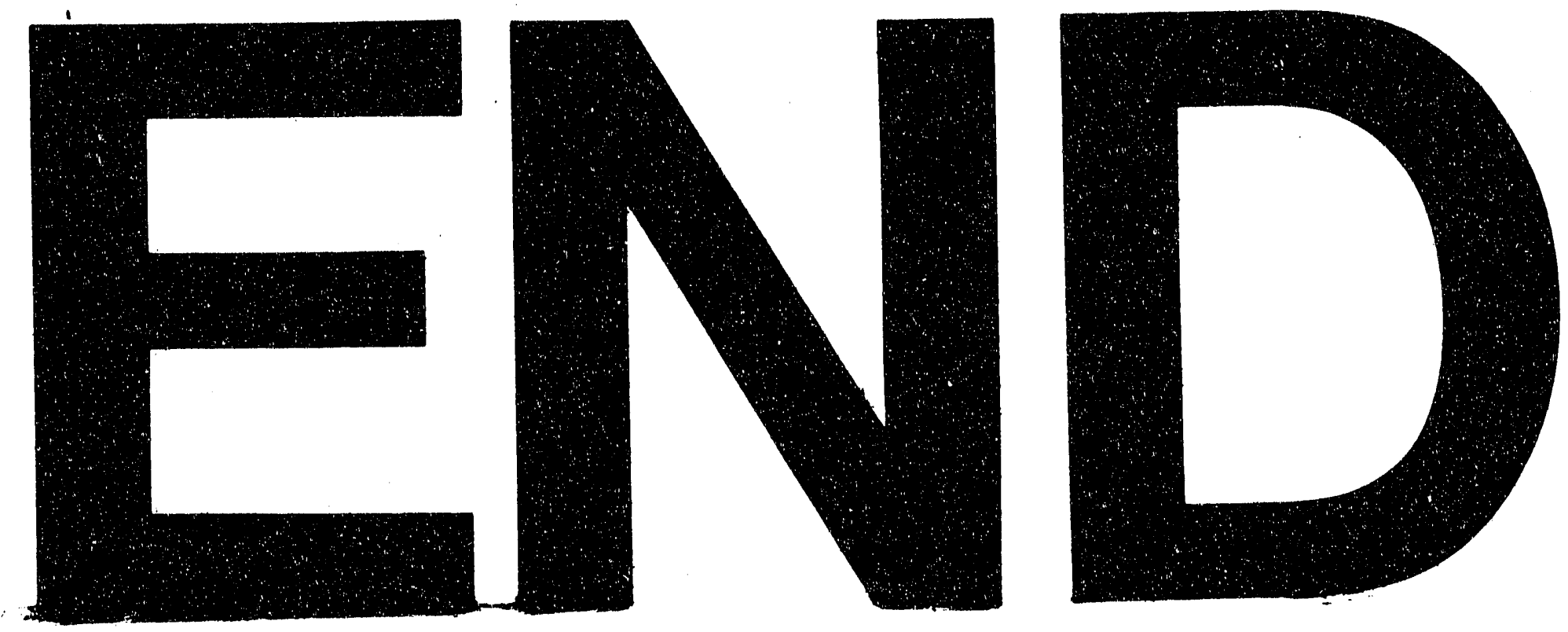

7
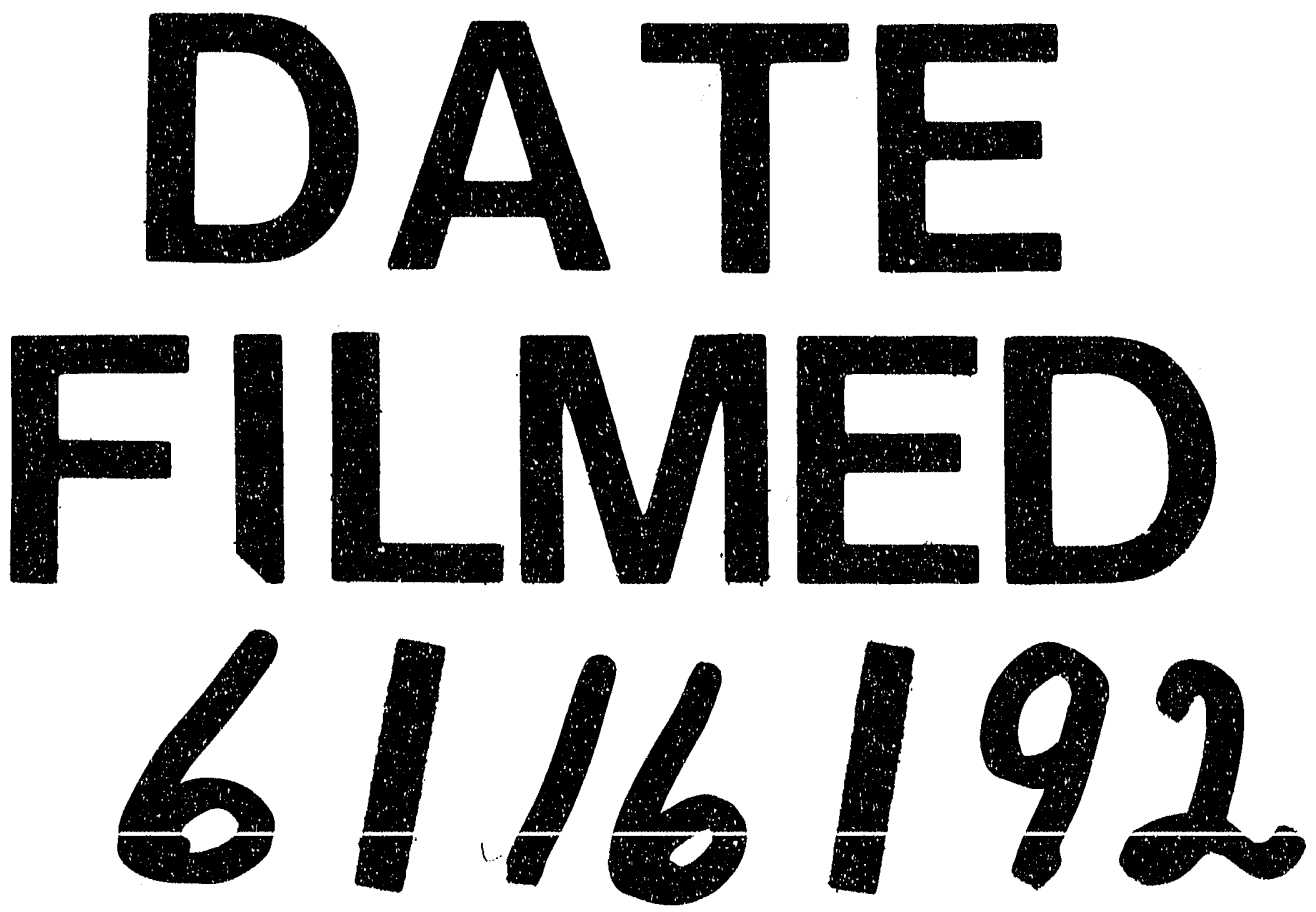


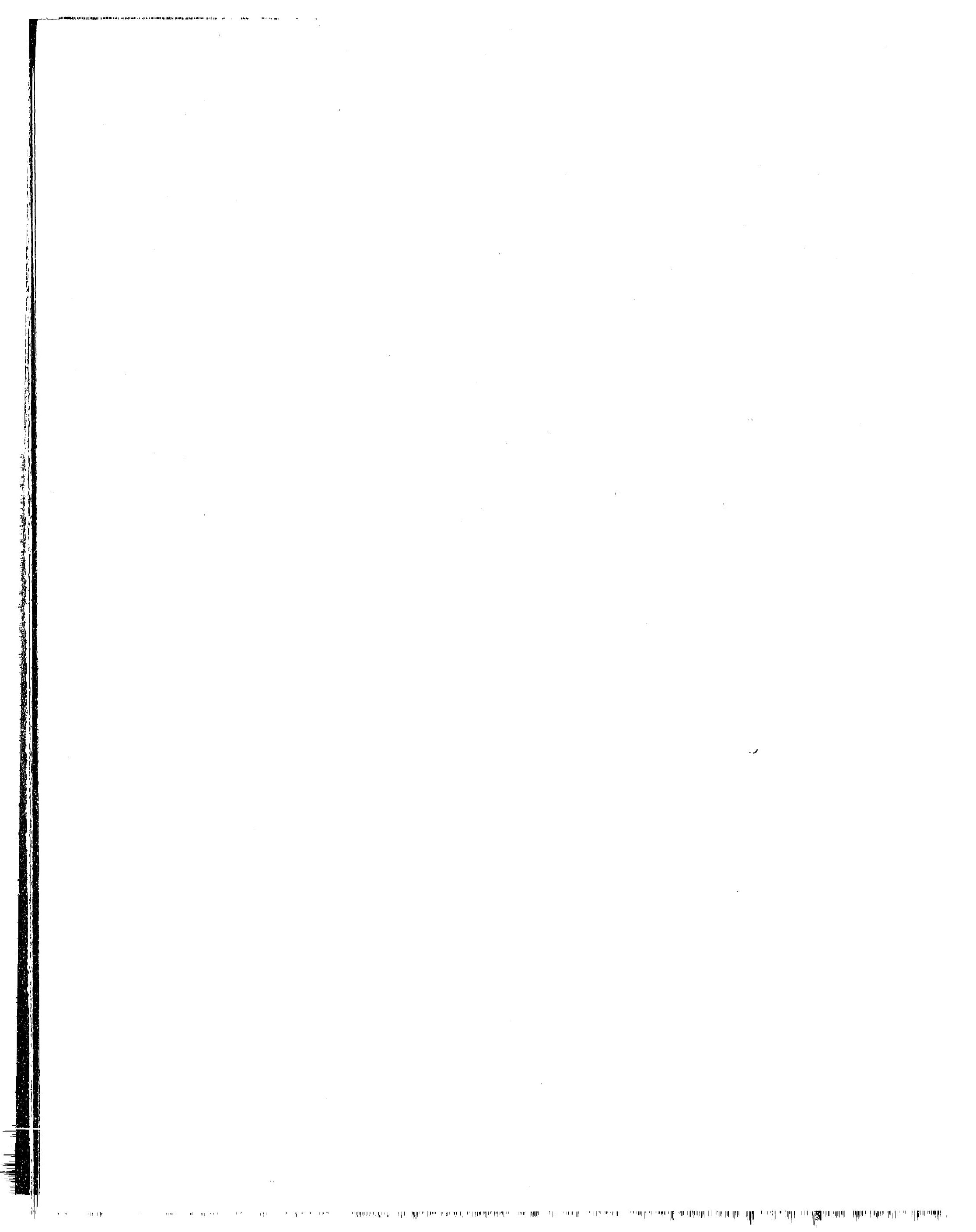

